TRANSACTIONS OF THE

AMERICAN MATHEMATICAL SOCIETY

Volume 354, Number 5, Pages 1705-1748

S 0002-9947(02)02948-3

Article electronically published on January 11, 2002

\title{
ON LOOP SPACES OF CONFIGURATION SPACES
}

\author{
F. R. COHEN AND S. GITLER
}

\begin{abstract}
This article gives an analysis of topological and homological properties for loop spaces of configuration spaces. The main topological results are given by certain choices of product decompositions of these spaces, as well as "twistings" between the factors. The main homological results are given in terms of extensions of the "infinitesimal braid relations" or "universal YangBaxter Lie relations".
\end{abstract}

\section{INTRODUCTION}

The purpose of this article is to describe properties of loop spaces of classical configuration spaces. The referee of this paper has requested that additional background as well as motivation for this paper be included. This additional information, as well as further applications and problems, will be given in the Introduction and section 2 of this paper.

The classical configuration space of a space $M, F(M, k)$, is the space of ordered $k$-tuples of distinct points in the product space $M^{k}$. In case $M$ is a manifold, the homology of the loop space for many configuration spaces is determined here. In contrast, the homology of the configuration space itself is not yet well-understood.

For example, if $M$ is given by a product of at least two spheres of dimension greater than zero, the integer homology of the configuration space is not understood at present. Some cases for which the homology is understood are given when $M=$ $N \times \mathbb{R}^{1}$ for a manifold $N$ [4, 9], while some beautiful general results in characteristic zero are given in 23] 29. Further connnections to function spaces are given in [1].

On the other hand, the homology of the loop space of the configuration space is frequently given in terms of Hopf algebras which arise in other contexts. The cases for which $M$ is a product of spheres, as well as many other manifolds, are included. A particular type of graded Lie algebra described in the next two paragraphs is central to these results.

Consider a Lie algebra which is universal for the (graded) "infinitesimal braid relations" or the "Yang-Baxter Lie algebra relations" as in [21, 22, 13, 15]. That is the largest Lie algebra over a fixed commutative ring $R$ for which the "infinitesimal braid relations" are satisfied. These relations also appear as special cases in the Vassiliev invariants of braids 22, 6]. They also arise in the study of the $K Z$ (Knizhnik-Zamolodchikov) equations as integrability conditions for certain flat

Received by the editors October 12, 1999 and, in revised form, September 1, 2001.

2000 Mathematics Subject Classification. Primary 20F14, 20F36, 52C35, 55P35, 14 D99.

Key words and phrases. Braid groups, configuration spaces, descending central series, loop spaces.

The authors were partially supported by the National Science Foundation Grant number 9704410. 
bundles [2], as well as in work of Kohno [21, 22], and Drinfel'd [11, 12] on the Kohno-Drinfel'd monodromy theorem [2].

The "universal example" of such a Lie algebra, $\mathcal{L}_{k}(q)$, is the free (graded) Lie algebra generated by elements $B_{i, j}$ of degree $q, k \geq i>j \geq 1$, modulo the graded infinitesimal braid relations:

(i): $\left[B_{i, j}, B_{s, t}\right]=0$ if $\{i, j\} \cap\{s, t\}=\varnothing$,

(ii): $\left[B_{i, j}, B_{i, t}+(-1)^{q} B_{t, j}\right]=0$ if $1 \leq j<t<i \leq k$, and

(iii): $\left[B_{t, j}, B_{i, j}+B_{i, t}\right]=0$ if $1 \leq j<t<i \leq k$.

T. Kohno 21] gives a slightly different description of these relations as follows: Introduce new generators $B_{j, i}$ of degree $q, k \geq i>j \geq 1$, with the relations $B_{i, j}=(-1)^{q} B_{j, i}$. Then Kohno's description of the above relations simplifies to (i) and (ii) above with distinct $i, j$, and $t$.

In addition, a second Lie algebra occurs which is an extension of the above Lie algebra $\mathcal{L}_{k}(q)$ by a quotient of the coproduct of $(1) \mathcal{L}_{k}(q)$ with (2) the $k$-fold sum $\bigoplus_{k} L$ of a given Lie algebra $L$ (which is required to satisfy certain freeness conditions specified below). This extension denoted $\mathcal{L}_{k}(q) \prec L$ is defined precisely in section 4. The extension arises in descriptions of the homology for the loop space of configuration spaces for more general manifolds, and reflects the underlying topology of the manifold. Furthermore, the Lie algebras $\mathcal{L}_{k}(q) \prec L$ satisfy additional universal relations which will be called the "extended infinitesimal braid relations", and which are given in the next paragraph as well as in section 4 .

For a fixed element $x$ in $L$, let $x_{i}$ denote $x$ in the $i$-th coordinate of the $k$-fold sum $\bigoplus_{k} L$. The "extended infinitesimal braid relations" in $\mathcal{L}_{k}(q) \prec L$ are specified as follows:

(iv): $\left[B_{i, j}, x_{s}\right]=0$ if $\{i, j\} \cap\{s\}=\varnothing$, and

(v): $\left[B_{i, j}, x_{i}+x_{j}\right]=0$.

The Lie algebra which is "universal" for these relations is discussed before Theorem 2.10 and in section 4 here.

The results in Theorem 2.3 here give that the loop space homology of ordered configurations in a Euclidean space is the universal enveloping algebra of the "universal Yang-Baxter Lie algebra" alluded to above. The loop space homology of configuration spaces for manifolds which are not closed is given in Theorem 2.4. In favorable cases, the loop space homology of the configuration space is given by the universal enveloping algebra for a Lie algebra which satisfies the "extended infinitesimal braid relations".

Some cases of manifolds $M$ for which the loop space homology of their configuration spaces admit "clean" descriptions are listed next. These manifolds $M$ include those which

1. have a Euclidean factor,

2. are Lie groups of rank at least 3 , or

3. have more than 2 factors in a non-trivial product decomposition.

Modifications required for other cases are considered below where information concerning the tangent bundle of the manifold gives information about the precise nature of the loop space homology for the loop space of the configuration space for the manifold (Theorem 2.10). These required modifications are described next.

To analyze the structure of these loop spaces, a further analogue of "braiding " is required. A braid with $k$ strands may be described loosely as $k$ paths of points in $\mathbb{R}^{2}$ parametrized by a time parameter $t$ in the unit interval $[0,1]$, and where the 
points are not allowed to collide at any time $t$. A picture of the associated braid is given by the graph of the paths in question. A braid is "pure" if the initial point of each path is also the terminal point. Thus a pure braid may be thought of as a loop in the configuration space of $k$ points in $\mathbb{R}^{2}$.

Similarly, the loop space of $F(M, k)$ can be thought of as the space of paths of $k$ particles in $M$ where

1. the particles end at the positions where they start, and

2. the particles are not allowed to collide at any time $t$.

An analogous kind of "braiding" is given by paths of disjoint subspaces parametrized by time in $[0,1]$. Paths of certain choices of subspaces in addition to paths of particles are considered here. This vague description is translated to a precise description of the notion of a "braidable" manifold in Definition 2.5.

One of the theorems proven below gives an analysis of the homology of the loop space for the configuration spaces of manifolds which are both "braidable", and for which certain characteristic classes for their tangent bundle vanish. In these cases, the loop space homology is the universal enveloping algebra of a Lie algebra which satisfies the "extended infinitesimal braid relations" given above.

Examples of manifolds which satisfy the definition of "braidable" are given by $\mathbb{R} \times M$ for manifolds $M$, or $S U(n)$ for $n>3$. Examples of manifolds which do not satisfy the definition of "braidable" are given by products of exactly 2 spheres, $S U(3)$, or $S p(2)$. The homology of the loop spaces of configurations spaces for manifolds which are not "braidable" have additional "twistings", while the "extended infinitesimal braid relations" frequently fail in these cases. In addition, the "twistings" in these cases are distinctly different, and more complicated in the case of $S U(3)$ in comparison to those for $S U(n)$ with $n$ greater than 3.

The results here may be regarded as homological versions of higher dimensional analogues of classical braids. Further homotopical versions are given in 8 . The elements $B_{i, j}$ which arise in the Lie algebras $\mathcal{L}_{k}(q)$ are in fact precise geometric analogues of representatives for the so-called "pure braids" which link strands $i$ and $j$. The maps which define the $B_{i, j}$ are obtained by embeddings of the $(n-1)$ sphere in products of Euclidean $n$-space which link the $i$ and $j$ coordinates, as is the case with pure braids in the case of $\mathbb{R}^{2}$. These maps were defined and used to analyze the cohomology of configuration spaces of points in a Euclidean space [4.

There is a further connection to Vassiliev invariants of braids which were determined in work of T. Kohno 22]. Namely, one of the results stated in 6] gives that the homology of the loop space for the configuration space of points in $\mathbb{R}^{4}$ is, as a Hopf algebra, isomorphic to the graded Hopf algebra given by the Vassiliev invariants obtained from horizontal chord diagrams with a degree shift. In addition, the results here apply to configuration spaces for more general manifolds, and admit further applications.

In the special case that the underlying manifold $M$ is $\mathbb{R}^{n}$, the work here also gives that two, apparently different, mathematical objects are isomorphic up to a trivial shift of degrees. One object is group theoretic, and is given by the Lie algebra obtained from the descending central series of a discrete group $G$ for which $G$ is the pure braid group. The second object is the Lie algebra of primitive elements in the homology of a torsion free connected Hopf algebra given by the homology of the loop space of a configuration space. An explanation of this structure, as well 
as connections to some other mathematical structures, is given in [8]. One such application is described in the next three paragraphs.

The results of this article provide the motivation and the information for the construction of other "braid-like" groups which are defined for any manifold of dimension at least 3, and which are analogous to the classical pure braid groups. More precisely, the pure braid group associated to a surface is usually given as the fundamental group of the configuration space for that surface. If the manifold is simply-connected and of dimension at least 3, then the fundamental group of the configuration space is trivial. Hence, the pure braid group defined in this way for manifolds of dimension at least 3 is trivial.

In [8], the computations here are used to define a "braid-like" group for any manifold. The resulting groups are non-trivial even for Euclidean spaces of dimensions greater than 2. For example, these "braid-like" groups are non-isomorphic if the dimensions of the Euclidean spaces differ. However, the $\mathrm{Mal}^{\prime}$ cev completions of these groups are isomorphic for all even dimensional Euclidean spaces. The elements $B_{i, j}$ alluded to above give generators for the "braid-like" group attached to any Euclidean space.

The " $k$-stranded braid-like" groups for an arbitrary space $M$ are defined in [8] as the group of coalgebra morphisms from a tensor algebra with a single generator in degree 1 to the homology of the loop space of the configuration space $F(M, k)$. Some properties of these groups are given next.

1. The groups are filtered (however, not by the descending series filtration), and the associated graded modules form a Lie algebra.

2. In case the underlying manifold is complex $n$-space, then this Lie algebra is the one above $\mathcal{L}_{k}(2 n-2)$.

3. In certain favorable cases for $M$, this Lie algebra is given by one which satisfies the "extended infinitesimal braid relations" given by $\mathcal{L}_{k}(q) \imath L$ for some choice of Lie algebra $L$.

In addition the loop spaces here provide crude approximations to spaces of curves in a manifold as follows. Consider the the space of continuous functions from a circle to a space $M$ together with the quotient "moduli space" obtained by factoring out the homeomorphisms of the source by the natural action on the function space. The resulting quotient may be thought of as the space of curves in $M$. However, the action of the homeomorphism group on the function space is usually not free.

In what follows below, $E G$ denotes Milnor's infinite join construction for a topological group $G$. Thus in favorable cases, there is a principal $G$-bundle

$$
E G \rightarrow B G
$$

where $B G$ denotes $E G / G$. One natural construction is to form the balanced product $E G \times{ }_{G} \Lambda M$, where $\Lambda M$ denotes the free loop space for $M$, the space of all continuous (unpointed) maps from $S^{1}$ to $M$, and $G$ is either $O(2), S O(2)$, the group of diffeomorphisms of $S^{1}$, or the group of homeomorphisms of $S^{1}$. The resulting object may be regarded as an approximation for the "moduli space" of curves on $M$ as it admits a singular fibration over this "moduli space" $E G \times_{G} \Lambda M \rightarrow G \backslash \Lambda M$.

The types of constructions considered here are targets of various embedding spaces. For example, let $\operatorname{Emb}\left(S^{1}, M\right)$ denote the space of embeddings of $S^{1}$ and $M$. Let $\zeta_{k}=e^{2 \pi i / 2^{k}}$, with $\pi_{k}$ generated by $\zeta_{k}$. Evaluating an embedding $f$ on the 
cyclic group $\pi_{k}$ results in a map

$$
\theta: \operatorname{Emb}\left(S^{1}, M\right) \rightarrow \Lambda\left(\lim _{2^{k}} F\left(M, 2^{k}\right)\right)
$$

which is $S O(2)$-equivariant.

As a special case for which $G$ is $S O(2)$, passage to quotients gives a natural map

$$
E S O(2) \times_{S O(2)} \operatorname{Emb}\left(S^{1}, M\right) \rightarrow E S O(2) \times_{S O(2)} \Lambda\left(\varliminf_{2^{k}} F\left(M, 2^{k}\right)\right) .
$$

The rational homology of the "layers" for the target,

$$
E S O(2) \times_{S O(2)} \Lambda F\left(M, 2^{k}\right),
$$

is given in terms of the cyclic homology of the enveloping algebra for the universal "Yang-Baxter Lie algebra" when $M$ is Euclidean space. The resulting orbit space can be thought of as the space of embeddings modulo rotations, and gives characteristic classes for the space of curves on $M$.

The homology of the loop space of a configuration space "fits" in several different contexts connected to embeddings, generalizations of braids in higher dimensions, and invariants of braids. That is part of the motivation for the work given here.

The loop space homology of configurations in a Euclidean space was first analyzed by E. Fadell and S. Husseini [13]. Their questions prompted the work here, as well as the work of M. Xicoténcatl on orbit configuration spaces for a manifold with a free action of a group [31. Betti numbers for the loop space homology of many configuration spaces are given by Y. Félix and J.-C. Thomas [17].

The organization of this article is as follows:

1: Introduction

2: Results

3: Proof of Theorem 2.1; splittings of loop spaces

4: The "universal Yang-Baxter Lie algebras"; Theorem 2.2

5: Homological calculations for $\mathbb{R}^{m}$

6: Enveloping algebras and the proof of 2.3

7: General twistings; proof of Theorem 2.4

8: Commutativity relations; proofs of Theorems 2.6 and 2.7

9: Proof of Theorem 2.8

10: Loop spaces of universal sphere bundles; proof of Theorem 2.9

11: Loop spaces of tangent bundles; commutativity relations Theorem 2.10

12: On the case of $M-$ \{point $\}$ in $M$; proof of Theorem 2.11

13: Proof of Theorem 2.12

14: On $\Omega(G / H)$

15: On $\Omega F(M, k)$ for $M=S U(n), S p(n)$, or $G / H$; proof of Theorem 2.13

16: Special cases; Theorem 2.14

\section{REsults}

Let $M$ denote a path-connected $m$-dimensional manifold without boundary; $M$ is sometimes required to be closed and sometimes not. Recall from [14 that the ordered configuration space is given by

$$
F(M, k)=\left\{\left(m_{1}, \ldots, m_{k}\right) \in M^{k} \mid m_{i} \neq m_{j} \text { if } i \neq j\right\} .
$$

The subject of this article is an analysis of the loop space homology of $F(M, k)$ as described next. 
The space of all continuous functions from $S^{1}$ to a space $X, \Lambda X$, is the unbased loop space. If $X$ has a base-point, $\Omega X$ is the subspace of $\Lambda X$ given by all pointed maps. There is a fibration $\Lambda X \rightarrow X$ with fibre $\Omega X$. The main subject of the work here is $\Omega X$.

If $M$ is the complement of a point in a manifold $M^{\prime}$, then $M$ is a punctured manifold and $M$ will be called a $p$-manifold. If the natural inclusion of $M-\{$ point in $M$ induces an epimorphism on the level of the homology of loop spaces with coefficients in a ring $R$ and both homology rings are $R$-torsion free, then $M$ will be called an $e R$-manifold. If the first coordinate projection map $\pi: F(M, k) \rightarrow M$ admits a cross-section up to homotopy, then $M$ will be called a $\sigma_{k}$-manifold. These definitions give useful general conditions for the work here.

The first result below gives product decompositions for loop spaces of many configuration spaces. These decompositions are not multiplicative. Most of the work of this article is addressing how these factors are twisted together. The next theorem follows immediately from the sections given in [14 together with standard homotopy theoretic properties where $Q_{i}$ denotes a set of $i$ distinct points $\left\{q_{1}, \ldots, q_{i}\right\}$ in $M$. The suspension of a space $M$ is denoted $\Sigma M$. The smash product of two pointed spaces $M \wedge N$ is the quotient of the product given by $M \times N / M \vee N$.

Theorem 2.1. $\quad$ 1. If $M$ is a $\sigma_{k}$-manifold, then there is a homotopy equivalence

$$
\Omega F(M, k) \longrightarrow \Omega M \times \Omega F\left(M-Q_{1}, k-1\right), \quad k \geq 2 .
$$

Furthermore, there are homotopy equivalences

$$
\Omega F\left(M-Q_{1}, k\right) \longrightarrow \prod_{i=1}^{k} \Omega\left(M-Q_{i}\right), \quad k \geq 1,
$$

for any manifold $M$ (without boundary).

2. If $m \geq 3$, there is a homotopy equivalence

$$
\Omega F\left(\mathbb{R}^{m}, k\right) \longrightarrow \prod_{i=1}^{k-1} \Omega\left(\bigvee_{i} S^{m-1}\right)
$$

3. If $M$ is a p-manifold of dimension at least 3, then there is a homotopy equivalence

$$
\Omega F(M, k) \longrightarrow(\Omega M)^{k} \times \Omega F\left(\mathbb{R}^{m}, k\right) \times \prod_{i=3 D 1}^{k-1} \Omega \Sigma\left[(\Omega M) \wedge\left(\Omega \bigvee_{i} S^{m-1}\right)\right]
$$

Furthermore, there is a choice of homotopy equivalence

$$
\Sigma \Omega F(M, k) \longrightarrow\left(\bigvee_{\substack{j_{\beta} \in J \\ i_{\alpha} \in I}} \Sigma^{j_{\beta}}(\Omega M)^{\left(i_{\alpha}\right)}\right) \vee\left(\bigvee_{\gamma \in K} S^{k_{\gamma}}\right)
$$

for some choices of index sets $I, J$ and $K$.

4. If $M=M_{1} \times M_{2}$, for non-empty manifolds $M_{i}, i=1,2$, of dimension at least one, then then there is a homotopy equivalence

$$
\Omega F(M, k) \longrightarrow \Omega(M) \times \Omega F\left(M-Q_{1}, k\right) .
$$


The utility of this theorem is that it gives the additive structure for the homology of the loop space of the configuration space. If $M$ is a $p$-manifold of dimension at least 3, this answer depends only on the additive structure for the homology of the loop space of $M$, and the number of puncture points. The splittings above are not multiplicative and require more input from the topology of the manifold $M$. The way in which the homologies of these spaces are "twisted" together to give the multiplicative structure for the loop space is determined below. The answer is frequently given by the universal enveloping algebra of the Lie algebras described above, obtained from the extended infinitesimal braid relations.

The main work of this article is an analysis of the "twisting" which is the failure of multiplicativity for the product decompositions in the above theorem. The first such "twisting" is homological in nature and arises from the infinitesimal braid relations or "Yang-Baxter Lie algebra" relations. A description of this "twisting" requires the construction of a Lie algebra with certain universal properties.

Here, let $V_{n}(q)$ denote the free graded abelian group of rank $n$ which is concentrated in degree $q$ and has basis

$$
\left\{x_{n+1,1}, x_{n+1,2}, \ldots, x_{n+1, n}\right\} .
$$

Let $L\left[V_{n}(q)\right]$ denote the free graded Lie algebra generated by $V_{n}(q)$, and let

$$
\lambda: \bigoplus_{n=1}^{k-1} V_{n}(q) \longrightarrow \mathcal{G}
$$

be a homomorphism of graded abelian groups, where $\mathcal{G}$ is a graded Lie algebra. Let $B_{i, j}=\lambda\left(x_{i, j}\right)$. The (graded) infinitesimal braid relations or "Yang-Baxter Lie relations" are as follows [13], [21]:

1. $\left[B_{i, j}, B_{s, t}\right]=0$ if $\{i, j\} \cap\{s, t\}=\varnothing$,

2. $\left[B_{i, j}, B_{i, t}+(-1)^{q} B_{t, j}\right]=0$ for $1 \leq j<t<i \leq k$, and

3. $\left[B_{t, j}, B_{i, j}+B_{i, t}\right]=0$ for $1 \leq j<t<i \leq k$.

Let $\mathcal{L}_{k}(q)$ denote the free graded Lie algebra generated by $\bigoplus_{n=1}^{k-1} V_{n}(q)$ modulo the infinitesimal braid relations.

Theorem 2.2. If $\mathcal{G}$ is a graded Lie algebra and $\lambda: \bigoplus_{n=1}^{k-1} V_{n}(q) \rightarrow \mathcal{G}$ is a homomorphism of graded abelian groups such that $\lambda\left(x_{i, j}\right)$ satisfy the infinitesimal braid relations, then there is a unique homomorphism of graded Lie algebras

$$
g: \mathcal{L}_{k}(q) \longrightarrow \mathcal{G}
$$

which factors $\lambda$. Furthermore, there are homomorphisms of Lie algebras

$$
L\left[V_{n}(q)\right] \rightarrow \mathcal{L}_{k}(q)
$$

for $1 \leq n \leq k-1$ such that the induced homomorphism of graded abelian groups

$$
\bigoplus_{1 \leq n \leq k-1} L\left[V_{n}(q)\right] \rightarrow \mathcal{L}_{k}(q)
$$

is an isomorphism (of graded abelian groups).

The next theorem exhibits this twisting more topologically. Let PrimH denote the module of primitive elements in a connected graded Hopf algebra $H$. 
Theorem 2.3. If $m \geq 3$, the homology of $\Omega F\left(\mathbb{R}^{m}, k\right)$ is torsion free and there is an isomorphism of Lie algebras on the level of the module of primitives:

$$
\mathcal{L}_{k}(m-2) \rightarrow \operatorname{Prim}_{*}\left(\Omega F\left(\mathbb{R}^{m}, k\right) ; \mathbb{Z}\right) .
$$

Furthermore, the induced map on the level of universal enveloping algebras

$$
U \mathcal{L}_{k}(m-2) \rightarrow H_{*}\left(\Omega F\left(\mathbb{R}^{m}, k\right) ; \mathbb{Z}\right)
$$

is an isomorphism of Hopf algebras. If $E_{*}$ is any homology theory, then there is an isomorphism of algebras

$$
E_{*}(\text { point }) \otimes_{\mathbb{Z}} U \mathcal{L}_{n-1}(m-2) \rightarrow E_{*}\left(\Omega F\left(\mathbb{R}^{m}, n\right)\right) .
$$

If $M$ is a $p$-manifold, then these results for $\mathbb{R}^{m}$ imply information for $\Omega F(M, k)$. Fix an embedding $e: \mathbb{R}^{m} \subset M$, where $M$ is of dimension $m$ and $\mathbb{R}^{m}$ is the interior of a disk in $M$. There is an induced map

$$
F(e, k): F\left(\mathbb{R}^{m}, k\right) \rightarrow F(M, k) .
$$

Since $M$ is a $p$-manifold, there are sections

$$
\sigma_{i}: M-Q_{i} \rightarrow F\left(M-Q_{i}, k-i\right) .
$$

Regarding $M$ as homeomorphic to $M^{\prime}$-disk and using the sections $\sigma_{i}$, there are induced maps

$$
s_{i}: M \rightarrow F(M, k)
$$

given by the composite

$$
M \rightarrow M^{\prime}-\operatorname{disk} \subset M-Q_{i} \subset F\left(M-Q_{i}, k-i\right) \subset F(M, k) .
$$

The maps $s_{i}$ in the previous paragraph provide maps $\Omega\left(s_{i}\right): \Omega M \rightarrow \Omega F(M, k)$ that are used in the next theorem which gives "most" of the multiplicative structure for the homology of $\Omega F(M, k)$. When $m$ is any class in the loop space homology of $M$, write $m_{\ell}$ for the image $\left(\Omega s_{\ell}\right)_{*}(m)$.

Theorem 2.4. Let $M$ be a simply-connected $p$-manifold of dimension $m \geq 3$, and let $E_{*}$ be any homology theory which satisfies the strong form of the Künneth theorem for $(\Omega M)^{k}$. [That is, $E_{*}(\Omega M)^{k}$ is isomorphic to $\bigotimes_{E_{*}(\text { point })}^{k}\left(E_{*} \Omega M\right)$.]

1. An embedding $e: \mathbb{R}^{m} \rightarrow M$ induces a monomorphism

$$
E_{*} \Omega F\left(\mathbb{R}^{m}, k\right) \rightarrow E_{*} \Omega F(M, k) .
$$

2. $E_{*} \Omega F(M, k)$ is generated as an algebra by the images of

(a) $E_{*} \Omega F\left(\mathbb{R}^{m}, k\right)$, and

(b) $E\left(\Omega s_{i}\right)_{*}\left(E_{*} \Omega M\right)$ for $1 \leq i \leq k$.

3. The following relations are satisfied:

(i) the infinitesimal braid relations for the images of $B_{i, j}$ (the algebra generators for $E_{*} \Omega F\left(\mathbb{R}^{m}, k\right)$ specified in Theorem 2.3),

(ii) $\left[B_{i, j}, m_{\ell}\right]=0$ if $\ell \notin\{i, j\}$ and $m_{\ell}$ is in the image of $E_{*} \Omega\left(s_{\ell}\right)$,

(iii) $\left[B_{i, j}, m_{i}+m_{j}\right]=0$, where $m$ denotes a primitive element in the singular homology of $\Omega(M)$ with field coefficients whenever

(i) $\mathbb{F}=\mathbb{Z} / 2 \mathbb{Z}$ and $w_{m-1}(\tau)=0$, where $\tau$ is the unit sphere bundle in the tangent bundle for $M$, or

(ii) $\mathbb{F}=\mathbb{Z} / p \mathbb{Z}$ or $\mathbb{Q}$, where $p$ is an odd prime and the Euler class $e(\tau)$ is zero. 
The infinitesimal braid relations provide the initial input for the homological algebra extensions here which reflect the "twistings" between the factors described in Theorem 2.1. However, notice that the algebra extension given in Theorem 2.4 is not completely determined by the infinitesimal braid relations. The remaining relations required to solve this extension question are given by the commutation relations

1. $\left[B_{i, j}, m_{\ell}\right], \ell \in\{i, j\}$, and

2. $\left[m_{i}, m_{j}^{\prime}\right]$.

These additional relations determine the algebra structure completely for the homology of the loop space of a configuration space. The precise nature of these relations requires further geometrical input concerning the manifold $M$, and are the extensions of the infinitesimal braid relations.

It is the case that either $\left[B_{i, j}, m_{\ell}\right]$ or $\left[m_{i}, m_{j}^{\prime}\right]$ is sometimes zero and sometimes not. Additional topological information is required to determine the relations for these elements. It will be seen below that the value of the element $\left[B_{i, j}, m_{i}\right]$ is frequently non-zero when $M$ is a $p$-manifold. An explicit evaluation of this element in Theorem 2.9 follows from properties of the tangent bundle for $M$, a theorem of Ganea [18], and Theorem 2.1.

The relations for $\left[m_{i}, m_{j}^{\prime}\right]$ for $i>j$ are sometimes complicated and sometimes not. A general form of vanishing of $\left[m_{i}, m_{j}^{\prime}\right]$ for $i>j$ depends on a crude analogue of "general position", which itself is an analogue for braiding submanifolds of $M$ and is given next.

Definition 2.5. Let $M$ be a simply-connected manifold. Then $M$ is "braidable" if and only if

1. there exists a subspace $A$ of $M$ together with embeddings

$$
e_{i}: A \rightarrow M,
$$

for $i=1,2$, with disjoint images, such that

2. $e_{1}$ is isotopic to $e_{2}$, and

3. the induced maps $\Omega\left(e_{i}\right): \Omega(A) \rightarrow \Omega(M)$ (with respect to different basepoints) induce surjections in homology (with $R$-coefficients).

The utility of this definition is (i) it provides a method for deciding the relations $\left[m_{i}, m_{j}^{\prime}\right]$, and (ii) the definition is, in many cases, easily verified. In addition, the definition provides a homological measure of "braiding" the subspaces $A$ of a manifold $M$. Groups arising in this way are discussed in [8], where $A$ is "braided" in $M \times \mathbb{R}^{1}$ via a time parameter in much the same way as a set of points in the plane are braided in $\mathbb{R}^{2} \times \mathbb{R}^{1}$ via a time parameter. In particular, if $M$ is $\mathbb{R}^{2}$, this condition describes precisely the locus of two disjoint points moving through time.

Theorem 2.6. If $M$ is a p-manifold of dimension at least 3, and $M$ is braidable, then

$$
\left[m_{i}, m_{j}^{\prime}\right]=0 \quad \text { for all } i>j
$$

in $H_{*}(\Omega F(M, k) ; R)$.

Theorem 2.7. $\quad$ 1. The manifold $N \times \mathbb{R}^{1}$ is braidable when $A=N$.

2. If the dimension of $M$ is greater than twice the homological dimension of $M$, then $M$ is braidable. 
3. If $M_{i}$ are manifolds of dimension at least 1, then $M_{1} \times M_{2} \times M_{3}$ is braidable where $A=M_{1} \vee M_{2} \vee M_{3}$.

The next theorem gives that for $M=S U(3), S p(2)$, or the Stiefel manifold of orthonormal 2-frames in $\mathbb{R}^{n}, V_{n, 2}\left(\right.$ over $\left.\mathbb{F}_{2}\right)$, the elements $\left[m_{i}, m_{j}^{\prime}\right]$ are non-zero in $H_{*} \Omega F(M, k)$ for certain choices of $m_{i}$ and $m_{j}^{\prime}$. Thus these manifolds are not braidable, and the previous theorem does not apply in these cases. The requisite modifications are addressed next.

The cohomology rings of the manifolds $M$ given by $S U(3), S p(2)$, and $V_{n, 2}$ (over $\mathbb{F}_{2}$ with $n>3$ ) are exterior algebras, while their loop space homology is polynomial with generators denoted $x$ and $y$. These generators are used to describe the interaction between the elements $B_{i, j}$ and the loop space homology of $M$ in the next result, where $\mathbb{F}$ denotes a field. The main point of the "twisting" in these cases arises from non-zero Whitehead products in the homotopy groups of bouquets of spheres, and other spaces, as will be seen in the proof of the next theorem.

Theorem 2.8. There exist classes $m$ and $m^{\prime}$ in $H_{*}(\Omega M ; \mathbb{F})$, where

$$
\left[m_{i}, m_{j}^{\prime}\right] \neq 0
$$

in $H_{*}(\Omega F(M, k) ; \mathbb{F})$ for $k \geq i>j \geq 1$ in case $M$ is one of the following manifolds:

1. $S^{p} \times S^{q}-Q_{t}, t \geq 0, p, q \geq 2$,

2. $S U(3)-Q_{t}, t \geq 0$,

3. $S p(2)-Q_{t}, t \geq 0$,

4. $V_{n, 2}-Q_{t}, t \geq 0$, with $\mathbb{F}=\mathbb{F}_{2}$ (where $V_{n, 2}$ denotes the Stiefel manifold of ordered orthonormal 2-frames in $\mathbb{R}^{n}$ ) with $n>3$, and

5. $M$ is a connected sum $A \sharp B$ with $A$ or $B$ as in (1)-(4).

Furthermore, if $M=S U(3), S p(2)$, or $V_{n, 2}$, then $\left[m_{j}, m_{i}^{\prime}\right]$ is a non-zero scalar multiple of $B_{i, j}$ for $i>j$.

The final relation is that for $\left[B_{i, j}, m_{\ell}\right]$ with $\ell=i$, or $j$. The "universal example" for this relation will be given by the homology of the loop space for the universal $(n-1)$-sphere bundle with section, that is, $B S O(n-1)$. Introductory information concerning these bundles is listed next.

Recall that $E G$ is a contractible space on which the topological group $G$ acts properly discontinuously. Milnor's infinite join construction is an example of such an $E G$. In the case of interest here, $G$ is the Lie group $S O(n)$, and $E G$ can be taken to be the union of the Stiefel manifold of orthonormal $n$-frames in $\mathbb{R}^{n+k}$ as $k$ goes to infinity. The loop space considered next is that of $\operatorname{ESO}(n) \times_{S O(n-1)} S^{n-1}$ as described in section 10 .

The bundle $E S O(n) \times_{S O(n-1)} S^{n-1} \rightarrow B S O(n-1)$ with fibre $S^{n-1}$ has a crosssection. In addition, it is a classical fact that $\operatorname{ESO}(n) \times_{S O(n)} S^{n-1}$ is the sphere bundle in the standard disk bundle over $B S O(n)$, and is homotopy equivalent to $B S O(n-1)$ [27 26]. Since any multiplicative fibration with a cross-section is trivial, $\Omega\left(E S O(n) \times_{S O(n-1)} S^{n-1}\right)$ is homotopy equivalent to a product given by $S O(n-1) \times \Omega S^{n-1}$. However, this fibration fails to split multiplicatively, as will be seen by the homological computation given next.

To calculate $\left[B_{i, j}, m_{k}\right]$, it is useful to identify 3 distinct homology classes in $H_{*}\left(\Omega\left(E S O(n) \times_{S O(n-1)} S^{n-1}\right) ; \mathbb{F}\right)$ :

1. Let $\iota_{n-2}$ denote the fundamental cycle for $H_{n-2}\left(\Omega S^{n-1} ; \mathbb{F}\right)$. 
2. Let $x_{n-2}$ denote any class in $H_{n-2}\left(S O(n-1) ; \mathbb{F}_{2}\right)$ which is dual to the cohomology suspension of the Stiefel-Whitney class $w_{n-1}$.

3. If $n-1 \equiv 0(2)$, let $y_{n-2}$ denote any class dual to the cohomology suspension of the Euler class in $H^{n-1}(B S O(n-1) ; \mathbb{F})$.

Theorem 2.9. If $n>2$, the following formulas are satisfied in

$$
H_{*}\left(\Omega\left(E S O(n) \times_{S O(n-1)} S^{n-1}\right) ; \mathbb{F}\right) .
$$

(i) If $\mathbb{F}=\mathbb{F}_{2}$, then

$$
\left[\iota_{n-2}, x_{n-2}\right]=\iota_{n-2}^{2} .
$$

(ii) If $1 / 2 \in \mathbb{F}$ and $n-1 \equiv 0(2)$, then

$$
\left[\iota_{n-2}, y_{n-2}\right]=\lambda \iota_{n-2}^{2}
$$

for a choice of unit $\lambda$, and if $n-1 \equiv 1(2)$, then

$$
\left[\iota_{n-2}, x_{n-2}\right]=0 .
$$

The results in Theorems 2.4, 2.6 and 2.9 give a complete description of the algebra $H_{*}(\Omega F(M, k) ; \mathbb{F})$ for many cases. This structure is elucidated below, where the next theorem gives the loop space homology of many configuration spaces as a Hopf algebra. That description is given more cleanly by an algebraic construction which is given first.

Consider a graded Lie algebra $L$ over a field $\mathbb{F}$ together with the Lie algebra given by the $k$-fold sum of $L$ with itself, $L^{k}$. Let $x_{i}$ denote the element given by an element $x$ of $L$ in the $i$-th coordinate of $\bigoplus_{k} L$. Let $\mathcal{L}_{k}(q) \prec L$ denote the quotient of the coproduct of $\mathcal{L}_{k}(q)$ and $L^{k}$ modulo the extended infinitesimal braid relations as given above. One choice of graded Lie algebra $L$ is given by the module of primitive elements in the homology of a loop space, $\operatorname{Prim}_{*}(\Omega X ; \mathbb{F})$.

Theorem 2.10. Let $M$ be a simply-connected $p$-manifold of dimension $m \geq 3$ such that (1) $M$ is braidable,(2) the loop space homology of $M$ is primitively generated, and (3) either (a) $\mathbb{F}=\mathbb{Z} / 2 \mathbb{Z}$ with $w_{m-1}(\tau)=0$, where $\tau$ is the unit sphere bundle in the tangent bundle for $M$, or (b) $\mathbb{F}=\mathbb{Z} / p \mathbb{Z}$ or $\mathbb{Q}$, where $p$ is an odd prime and the Euler class $e(\tau)$ is zero. Then $H_{*}(\Omega F(M, k) ; \mathbb{F})$ is the associative algebra which is a primitively generated Hopf algebra generated by $B_{i, j}$ and $m_{\ell}$ with the additive homology specified by Theorem 2.4 subject precisely to the relations

1. the infinitesimal braid relations for the $B_{i, j}$,

2. $\left[B_{i, j}, m_{\ell}\right]=0$ if $\ell \notin\{i, j\}$,

3. $\left[B_{i, j}, m_{i}+m_{j}\right]=0$, and

4. $\left[m_{i}, m_{j}^{\prime}\right]=0$ for $i>j$.

Futhermore, the Lie algebra of primitive elements in $H_{*}(\Omega F(M, k) ; \mathbb{Q})$ is isomorphic to $\mathcal{L}_{k}(q)$ ? $\operatorname{PrimH}_{*}(\Omega M ; \mathbb{F})$ as a Lie algebra.

Some examples of Theorem 2.10 with $\mathbb{Q}$ coefficients are given by $M=S U(n)-Q_{t}$ for $n \geq 4$ and $t \geq 1$. The cases of $M=S U(3)$ or $S p(2)$ are different and are given below in Theorem 2.12 .

Hypotheses were made on the manifold $M$ in the above theorems. To return to the case of an arbitrary manifold, assume that $M$ is closed. By [16], the natural inclusion $M-\{$ point $\} \subset M$ induces a surjection in loop-space homology with rational coefficients provided $M$ is 1-connected with at least two independent generators for its rational cohomology ring. Thus in these cases $M$ is an $e R$-manifold for $R=\mathbb{Q}$. 
Y. Félix and J.-C. Thomas [17] use this property to obtain results very similar to the next theorem.

Theorem 2.11. If $M$ is an eR-manifold, then there is a short exact sequence of Hopf algebras

$$
1 \rightarrow H_{*}\left(\Omega F\left(M-Q_{1}, k-1\right) ; R\right) \rightarrow H_{*}(\Omega F(M, k) ; R) \rightarrow H_{*}(\Omega M ; R) \rightarrow 1 .
$$

Furthermore, $M-Q_{i}, i \geq 1$, is an eR-manifold, and thus $H_{*}(\Omega F(M, k) ; R)$ is isomorphic to

$$
\bigotimes_{i=0}^{k-1} H_{*}\left(\Omega\left(M-Q_{i}\right) ; R\right)
$$

as a graded $R$-module, and the natural inclusion $M-\{$ point $\} \subset M$ induces a surjection

$$
H_{*}\left(\Omega F\left(M-Q_{1}, k\right) ; R\right) \rightarrow H_{*}(\Omega F(M, k) ; R) .
$$

Notice that if $M$ is an $e R$-manifold, then one might regard $M-Q_{1}$ as "the universal example" for the loop space homology of configuration spaces. This fact gives some of the motivation for restricting to $p$-manifolds in many of the previous theorems. Examples of $e R$-manifolds are given next.

Theorem 2.12. 1 . If $R=\mathbb{Q}, M$ is simply-connected, and the ring $H^{*}(M ; \mathbb{Q})$ is not monogenic, then $M$ is an eR-manifold [16].

2. If $M=M_{1} \times M_{2}$ with dimension $\left(M_{i}\right) \geq 1$, and $R$ is a field, then $M$ is an eR-manifold.

3. The connected sum of simply-connected manifolds at least one of which is an $e R$-manifold is again an eR-manifold when $R=\mathbb{Q}$.

4. Certain choices of homogeneous spaces $G / H$ are eR-manifolds (as given in section 12).

5. Spheres and projective spaces are not eR-manifolds.

The next theorem describes the loop space homology for configuration spaces of $S U(n)$ and $S p(n-1)$ when coefficients are taken in the rational numbers. The results for the cases $n=3$ and $n \geq 4$ are distinctly different. In this theorem, the classes of degree $2 i$ given by $x_{i}$ for $n-1>i>0$ are the standard algebra generators for the loop space homology of $S U(n)$, while in the case of $S p(n-1)$ the $x_{i}$ are of degree $4 i-2$. These classes arise from the homology of complex projective spaces in the case of $S U(n)$ or quasi-projective spaces in case of $S p(n-1)$. Write $x_{i}(t)$ for $\left(\Omega s_{t}\right)_{*}\left(x_{i}\right)$ in the theorem below, where the $s_{t}$ are given immediately before Theorem 2.4 of this section. Similar results hold for certain choices of homogeneous spaces and are listed in Theorem 15.2.

Theorem 2.13. Let $G$ be a connected Lie group. If all loop spaces in this theorem are localized at the rational numbers, then there is a homotopy equivalence

$$
\Omega F(G, k) \longrightarrow(\Omega G)^{k} \times \Omega F\left(\mathbb{R}^{m}, k\right) \times \prod_{i=1}^{k-1} \Omega \Sigma\left[(\Omega G) \wedge\left(\Omega \bigvee_{i} S^{m-1}\right)\right]
$$

If $G=S U(n)$ or $S p(n-1)$, the relations for the homology of these loop spaces are specified in the proofs of Theorems 2.4 and 2.7 as follows:

1. the infinitesimal braid relations are satisfied for all $B_{i, j}$ for $k \geq i>j \geq 1$,

2. $\left[B_{i, j}, m_{\ell}\right]=0$ for $\ell \notin\{i, j\}$, 
3. if $G=S U(2)$ or $G=S p(1)$, then the homology ring of $\Omega F(G, k)$ is isomorphic to that of $\Omega(G) \times \Omega F\left(\mathbb{R}^{m}, k-1\right)$ as a Hopf algebra,

4. if $G=S U(3)$ or $G=S p(2)$, then $\left[x_{2}(i), x_{4}(j)\right]=B_{i, j}$ for $i>j$,

5. if $G=S U(n)$ or $G=S p(n-1)$ for $n \geq 4$, then $\left[x_{2 r}(i), x_{2 s}(j)\right]=0$ for all $i, j, r$, and $s$, and

6. $\left[B_{i, j}, m_{i}+m_{j}\right]=0$.

This theorem demonstrates some of the "twisting" between the loop space of the underlying manifold and the infinitesimal braid relations for $S U(n)$ or $S p(n-1)$. The cases of real, complex, and quaternionic projective spaces are studied by $\mathrm{M}$. Xicoténcatl [31. The construction of "orbit configuration spaces" is central to these cases. Some of Xicoténcatl's results are described here and in section 16 for completeness.

Let $G$ act on a space $Y$ with $Y \rightarrow Y / G$ a principal $G$-bundle. Then there is a principal $G^{k}$-bundle

$$
F_{G}(Y, k) \rightarrow F(Y / G, k)
$$

where $F_{G}(Y, k)=\left\{\left(y_{1}, \ldots, y_{k}\right) \in Y^{k} \mid y_{i} G \cap y_{j} G=\varnothing\right.$ if $\left.i \neq j\right\}$. Further, in the case of the above fibrations, he obtains the following splitting result in [31].

Theorem 2.14. There are homotopy equivalences

$$
\begin{aligned}
& \Omega F\left(\mathbb{R P}^{n}, k\right) \rightarrow(\mathbb{Z} / 2)^{k} \times \Omega F_{\mathbb{Z} / 2 \mathbb{Z}}\left(S^{n}, k\right), \\
& \Omega F\left(\mathbb{C P}^{n}, k\right) \rightarrow\left(S^{1}\right)^{k} \times \Omega F_{S^{1}}\left(S^{2 n+1}, k\right), \\
& \Omega F\left(\mathbb{H} \mathbb{P}^{n}, k\right) \rightarrow\left(S^{3}\right)^{k} \times \Omega F_{S^{3}}\left(S^{4 n+1}, k\right) .
\end{aligned}
$$

Other natural examples abound, such as the space of complex monic polynomials of degree $n$ where the difference of any two roots lies outside of a fixed lattice $\mathbb{Z}+\omega \mathbb{Z}$ in $\mathbb{C}$. These spaces, their cohomology, and their connections to modular forms will are addressed elsewhere [31, 3, 10].

\section{Proof of Theorem 2.1; SPlittings of LOOP SPACES}

Recall that if $p: E \rightarrow B$ is the projection map in a fibre bundle with the inclusion of the fibre $i: F \rightarrow E$ such that $p$ supports a cross-section $\sigma$, then $\Omega E$ is homotopy equivalent to $\Omega B \times \Omega F$. A choice of equivalence is gotten by the composite

$$
\Omega B \times \Omega F \stackrel{\Omega(\sigma) \times \Omega i}{\longrightarrow} \Omega E \times \Omega E \stackrel{\text { multiply }}{\longrightarrow} \Omega E .
$$

If $M$ is a $\sigma_{k}$-manifold, then the first coordinate projection $\pi: F(M, k) \rightarrow M$ is the projection map in a fibre bundle with fibre $F\left(M-Q_{1}, k-1\right)$ [14], and $\pi$ supports a section. Finally, the first coordinate projection map $\pi: F\left(M-Q_{i}, j\right) \rightarrow M-Q_{i}$ admits a section for all $i \geq 1$. Thus part (1) follows.

To prove part (2), notice that $\mathbb{R}^{m}-Q_{i}$ is homotopy equivalent to $\bigvee_{i} S^{m-1}$. Thus part (2) follows at once from (1).

Part (3) follows by induction on $k$. It is clear in case $k=1$. Assume the result for $k=q-1$ and check it for $k=q$. By [14 there is a fibre bundle with section

$$
F(M, q) \rightarrow F(M, q-1)
$$

with fibre $M-Q_{q-1}$, because $M$ is a $p$-manifold. Thus $\Omega F(M, q)$ is homotopy equivalent to the product

$$
\Omega F(M, q-1) \times \Omega\left(M-Q_{q-1}\right) .
$$


Notice that if $M$ is a $p$-manifold, then $\Omega F(M, q-1)$ satisfies the inductive hypothesis and so there exist homotopy equivalences

$$
\Omega\left(M-Q_{q-1}\right) \rightarrow \Omega\left(M \vee_{q-1} S^{m-1}\right)
$$

and

$$
\Omega\left(M \bigvee_{q-1} S^{m-1}\right) \rightarrow \Omega M \times \Omega\left(\bigvee_{q-1} S^{m-1}\right) \times \Omega \Sigma\left[\Omega M \wedge \Omega\left(\bigvee_{q-1} S^{m-1}\right)\right]
$$

by a crude form of the Hilton-Milnor theorem [24, 19, 25, 30, 18. Since $\Omega F\left(\mathbb{R}^{m}, q\right)$ is homotopy equivalent to $\Omega F\left(\mathbb{R}^{m}, q-1\right) \times \Omega\left(\vee_{q-1} S^{m-1}\right)$, the product decomposition in part (3) follows.

The decomposition for $\Sigma \Omega F(M, k)$ follows at once from the fact that $\Omega\left(\bigvee_{i} S^{m-1}\right)$ is homotopy equivalent to an infinite weak product of loop spaces of spheres if $i \geq 2$, and that $\Sigma \Omega S^{k+1}$ is homotopy equivalent to the wedge $\Sigma \bigvee_{j \geq 1} S^{j k}$.

Notice that if $M$ is a $p$-manifold, the additive structure for the loop space homology of $F(M, k)$ only depends on (1) the dimension of $M,(2)$ the loop space homology of $M$, and (3) $k$. The multiplicative structure requires more topology.

Furthermore, notice that the above decomposition gives a choice of homotopy equivalence

$$
(\Omega M)^{k} \times \Omega F\left(\mathbb{R}^{m}, k\right) \times \prod_{i=3 D 1}^{k-1} \Omega \Sigma\left[(\Omega M) \wedge \Omega\left(\bigvee_{i} S^{m-1}\right)\right] \rightarrow \Omega F(M, k)
$$

with some additional properties. This equivalence is a product of 3 maps, given by

$$
\begin{aligned}
& \alpha: \Omega(M)^{k} \rightarrow \Omega F(M, k), \\
& \beta: \Omega F\left(\mathbb{R}^{m}, k\right) \rightarrow \Omega F(M, k), \\
& \gamma: \prod_{i=1}^{k-1} \Omega \Sigma\left[(\Omega M) \wedge \Omega\left(\bigvee_{i} S^{m-1}\right)\right] \rightarrow \Omega F(M, k) .
\end{aligned}
$$

The second map $\beta$ is induced by the inclusion of $\mathbb{R}^{m}$ in $M$ as the interior of a fixed disk. The third map $\gamma$ is given by a product of $k-1$ maps, each of which is obtained by Whitehead products as described next.

Consider the inclusion $X \vee Y \subset X \times Y$ for pointed and path-connected spaces. The homotopy fibre of this inclusion is $\Sigma[(\Omega X) \wedge(\Omega Y)]$, and the map $\lambda$ : $\Sigma[(\Omega X) \wedge(\Omega Y)] \rightarrow X \vee Y$ is the Whitehead product of the two evaluation maps $\Sigma \Omega Z \rightarrow Z$ as described in [18.

Since this choice of equivalence will be used later, further details are given here.

Theorem 3.1. If $M$ is a p-manifold of dimension at least 3, there are loop maps

1. $\Omega\left(s_{i}\right): \Omega M \rightarrow \Omega F(M, k), 1 \leq i \leq k$,

2. $\Omega e: \Omega F\left(\mathbb{R}^{m}, k\right) \rightarrow \Omega F(M, k)$,

3. $\Omega \gamma_{i}: \Omega \Sigma\left[(\Omega M) \wedge \Omega\left(\bigvee_{i} S^{m-1}\right)\right] \rightarrow \Omega F(M, k), 1 \leq i \leq k$, such that the product of the maps given by

$$
\Theta=\left(\Omega s_{1} \times \cdot \times s_{k}\right) \times(\Omega e) \times\left(\Omega \gamma_{1} \times \cdots \times \Omega \gamma_{k}\right)
$$

gives a map

$$
(\Omega M)^{k} \times \Omega F\left(\mathbb{R}^{m}, k\right) \times \prod_{i=1}^{k} \Omega \Sigma\left[(\Omega M) \wedge \Omega\left(\bigvee_{i} S^{m-1}\right)\right] \rightarrow \Omega F(M, k)
$$

which is a homotopy equivalence. 
Proof. If $k=1, s_{1}$ is the identity and $\gamma_{1}$ is the trivial map. Assume that the maps have been given for $k<q$ so that the theorem has been satisfied.

Since $M$ is a $p$-manifold, the fibration $F(M, q) \rightarrow F(M, q-1)$ has a section with the fibre $M-Q_{k-1}$. Let $i: M-Q_{k-1} \rightarrow F(M, q)$ be the inclusion of the fibre. By the previous remarks, it suffices to exhibit $s_{q}$ and $\gamma_{q}$.

Again, since $M$ is a $p$-manifold, $M-Q_{q-1}$ is homotopy equivalent to $M \vee$ $\left(\bigvee_{q-1} S^{m-1}\right)$. The maps $s_{q}$ have been defined before the statement of Theorem 2.4 in section 2 and are given by the composite

$$
M \longrightarrow M \vee\left(\bigvee_{q-1} S^{m-1}\right) \stackrel{\phi}{\longrightarrow} M-Q_{q-1} \longrightarrow F(M, q)
$$

where $M \rightarrow M \vee\left(\bigvee_{q-1} S^{m-1}\right)$ is given by the natural map to $M$.

Define $\gamma_{q}$ to be the composite of the following three maps.

$$
\begin{gathered}
\gamma: \Sigma(\Omega M) \wedge \Omega\left(\bigvee_{q-1} S^{m-1}\right) \rightarrow M \vee\left(\bigvee_{q-1} S^{m-1}\right), \\
\phi: M \vee\left(\bigvee_{q-1} S^{m-1}\right) \rightarrow M-Q_{q-1},
\end{gathered}
$$

and

$$
i: M-Q_{q-1} \rightarrow F(M, q) .
$$

Notice that there is a homotopy commutative diagram

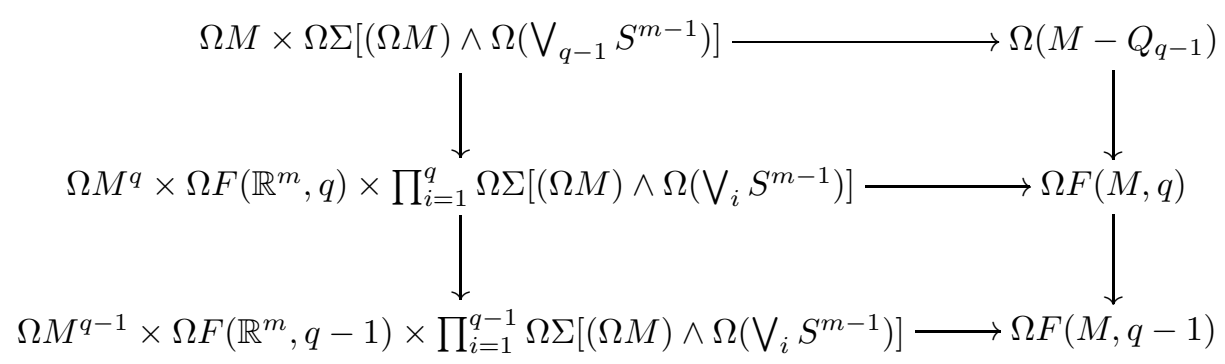

where the vertical arrows give fibrations with equivalences on the base and fibre. The third part of the theorem follows.

To prove the fourth part of the theorem, first consider two non-empty connected manifolds $M_{i}$, each of dimension at least one. The wedge $M_{1} \vee M_{2}$ is the subspace of the product $M_{1} \times M_{2}$ specified by the points $\left(m_{1}, p_{2}\right)$, and $\left(p_{1}, m_{2}\right)$ with $p_{i}$ a fixed point in $M_{i}$, and $m_{j}$ arbitrary in $M_{j}$. Choose $k$ distinct points $q_{1}, \cdots, q_{k}$ in $M_{1} \times M_{2} \backslash M_{1} \vee M_{2}$. Notice that there is a map $\sigma: M_{1} \vee M_{2} \rightarrow F(M, k)$ defined on points by the formula $\sigma(x)=\left(x, q_{2}, q_{3}, \cdots, q_{k}\right)$.

Notice that the composite of $\sigma$ with the first coordinate projection map is given by the natural inclusion $M_{1} \vee M_{2} \rightarrow M_{1} \times M_{2}$. Thus, after looping, $\Omega\left(M_{1} \times M_{2}\right)$ is a retract of $\Omega(F(M, k))$. The theorem follows.

\section{The "Universal Yang-BaXter Lie Algebra"; Theorem 2.2}

Let $\mathcal{L}_{k}(q)$ denote the Lie algebra constructed in section 2 after the statement of Theorem 2.1. That is, $\mathcal{L}_{k}(q)$ is the quotient of the free graded Lie algebra $L\left[\bigoplus_{n=1}^{k-1} V_{n}(q)\right]$ modulo the (graded) infinitesimal braid relations (or Yang-Baxter 
Lie relations) [13], 21], [15. That is, $\bigoplus_{n=1}^{k-1} V_{n}(q)$ has a basis $\left\{B_{i, j} \mid k \geq i>j \geq 1\right\}$ with relations in $\mathcal{L}_{k}(q)$ given by

1. $\left[B_{i, j}, B_{s, t}\right]=0$ if $\{i, j\} \cap\{s, t\}=\varnothing$,

2. $\left[B_{i, j}, B_{i, t}+(-1)^{q} B_{t, j}\right]=0$ for $1 \leq j<t<i \leq k$, and

3. $\left[B_{t, j}, B_{i, j}+B_{i, t}\right]=0$ for $1 \leq j<t<i \leq k$.

These relations were given by Kohno 21] in a slighty different formulation. As stated in the introduction, make the convention that $B_{i, j}=(-1)^{q} B_{j, i}$ for $1 \leq j<$ $i \leq k$. With $q=1$, the relations given by Kohno are identical with those above.

Given any homomorphism of graded abelian groups $f: \bigoplus_{n=1}^{k-1} V_{n}(q) \rightarrow \mathcal{G}$, where $\mathcal{G}$ is a graded Lie algebra, such that $f\left(B_{i, j}\right)$ satisfy relations (1), (2), and (3), there is a unique morphism of Lie algebras $\lambda: \mathcal{L}_{k}(q) \rightarrow \mathcal{G}$ which factors $f$. The main structure theorem for $\mathcal{L}_{k}(q)$ is as follows.

Theorem 4.1. $\quad$ 1. If $k \geq 2$, then $L\left[V_{k-1}(q)\right]$ is a Lie ideal in $\mathcal{L}_{k}(q)$ and there is a short exact sequence of Lie algebras

$$
0 \rightarrow L\left[V_{k-1}(q)\right] \rightarrow \mathcal{L}_{k}(q) \rightarrow \mathcal{L}_{k-1}(q) \rightarrow 0
$$

which is split as Lie algebras.

2. If $k=2$, then $\mathcal{L}_{2}(q)$ is isomorphic to the free Lie algebra $L\left[V_{1}(q)\right]$.

3. The Lie algebra $\mathcal{L}_{k}(q)$ is filtered by $F_{s} \mathcal{L}_{k}(q)$, the Lie sub-algebra generated by $B_{i, j}$ for $i \leq s$. The associated graded $E_{*}^{0}$ is isomorphic to

$$
\bigoplus_{s=1}^{k-1} L\left[V_{s}(q)\right]
$$

with $E_{s}^{0}=L\left[V_{s}(q)\right]$ as a graded abelian group.

Proof. The infinitesimal braid relations directly give that $L\left[V_{k-1}(q)\right]$ is a Lie ideal in $\mathcal{L}_{k}(q)$. Furthermore, the infinitesimal braid relations give no new relations in $L\left[V_{k-1}(q)\right]$.

Next, consider the map of Lie algebras

$$
\pi: \mathcal{L}_{k}(q) \rightarrow \mathcal{L}_{k-1}(q)
$$

induced by

$$
\pi\left(B_{i, j}\right)= \begin{cases}B_{i, j} & \text { if } 2 \leq i \leq k-2 \\ 0 & \text { if } i=k-1\end{cases}
$$

Notice that $\pi$ is a well-defined morphism of Lie algebras, by inspection of the infinitesimal braid relations. Furthermore, $\pi$ is surjective and is split by $\sigma$, where

$$
\sigma\left(B_{i, j}\right)=B_{i, j}, \quad 2 \leq i \leq k-2 .
$$

The kernel of $\pi$ is the Lie ideal generated by $B_{k-1, j}, 1 \leq j \leq k-2$. By inspection of the infinitesimal braid relations, the element

$$
\left[B_{k-1, j}, B_{s, t}\right]
$$

lies in the image of $L\left[V_{k-1}(q)\right]$ for all $\{j, s, t\}$. Furthermore, there are no relations for $\left[B_{k-1, j}, B_{k-1, \ell}\right]$ except for the graded Jacobi identity and antisymmetry. Thus (1) $L\left[V_{k-1}(q)\right]$ is isomorphic to the kernel of $\pi$, and (2) there is a short exact sequence of Lie algebras

$$
0 \rightarrow L\left[V_{k-1}(q)\right] \rightarrow \mathcal{L}_{k}(q) \rightarrow \mathcal{L}_{k-1}(q) \rightarrow 0
$$


As a graded abelian group, $\mathcal{L}_{k}(q)$ is thus isomorphic to $\mathcal{L}_{k-1}(q) \oplus L\left[V_{k-1}(q)\right]$.

Filter $\mathcal{L}_{k}(q)$ by requiring $F_{s} \mathcal{L}_{k}(q)$ to be the sub-Lie algebra generated by $B_{i, j}$ for $s \geq i>j \geq 1$. By the above remarks the inclusion $F_{s-1} \mathcal{L}_{k}(q)$ in $F_{s} \mathcal{L}_{k}(q)$ is a split monomorphism of Lie algebras, with $E_{s}^{0}$ isomorphic to $L\left[V_{s}(q)\right]$ as graded abelian groups.

Next, consider the universal enveloping algebra of $\mathcal{L}_{k}(q), U \mathcal{L}_{k}(q)$.

Corollary 4.2. There is a split short exact sequence of algebras

$$
1 \rightarrow U L\left[V_{k-1}(q)\right] \rightarrow U \mathcal{L}_{k}(q) \rightarrow U \mathcal{L}_{k-1}(q) \rightarrow 1 .
$$

Thus $U \mathcal{L}_{k}(q)$ is isomorphic to

$$
U \mathcal{L}_{k-1}(q) \otimes U L\left[V_{k-1}(q)\right]
$$

as a right $U L\left[V_{k-1}(q)\right]$-module. Furthermore, $U \mathcal{L}_{k}(q)$ is isomorphic to

$$
\bigotimes_{n=1}^{k-1} T\left[V_{n}(q)\right]
$$

as graded abelian groups.

Proof. Since

$$
0 \rightarrow L\left[V_{k-1}(q)\right] \rightarrow \mathcal{L}_{k}(q) \rightarrow \mathcal{L}_{k-1}(q) \rightarrow 0
$$

is a split short exact sequence of Lie algebras, the first part follows [7, 25]. Since $U L[V]$ is isomorphic to the tensor algebra $T[V]$, the second part follows at once 25].

Fix a ring $R$ with identity. Since $\mathcal{L}(q)$ is a finitely generated free abelian group, $R \otimes_{\mathbb{Z}} \mathcal{L}_{k}(q)$ is a free left $R$-module. Furthermore, the natural map

$$
\mathcal{L}_{k}(q) \longrightarrow R \otimes_{\mathbb{Z}} \mathcal{L}_{k}(q)
$$

is a morphism of Lie algebras.

Given a Lie algebra $L$ over a field $\mathbb{F}$, consider the coproduct of Lie algebras

$$
\Upsilon(L)=\left(\mathbb{F} \otimes_{\mathbb{Z}} \mathcal{L}_{k}(q)\right) \amalg\left(\bigoplus_{k} L\right) .
$$

Definition 4.3. Let

$$
\mathcal{L}_{k}(q) \curlywedge L
$$

denote the quotient of $\Upsilon(L)$ modulo the Lie ideal generated by the elements

1. $\left[B_{i, j}, x_{t}\right]$ for $t \notin\{i, j\}$, and

2. $\left[B_{i, j}, x_{i}+x_{j}\right]$,

where $x_{t}$ denotes an element $x$ in $L$ considered as an element in the $t$-th summand of $L$.

The Lie algebra extensions given in Theorem 4.1 admit analogous decompositions via an analogous computation. The proof of the following theorem is analogous to that of Theorem 4.1.

Theorem 4.4. There is a split short exact sequence of Lie algebras

$$
0 \rightarrow L\left[V_{k-1}(q)\right] \amalg L \rightarrow \mathcal{L}_{k}(q) \curlywedge L \rightarrow \mathcal{L}_{k-1}(q) \curlywedge L \rightarrow 0 .
$$


Proof. Notice that there is a morphism of Lie algebras

$$
\rho: \mathcal{L}_{k}(q) \curlywedge L \rightarrow \mathcal{L}_{k-1}(q) \curlywedge L
$$

obtained by setting

1. $\rho\left(B_{i, j}\right)=B_{i, j}$ for $i<k$,

2. $\rho\left(B_{k, j}\right)=0$ for $1 \leq j<k$,

3. $\rho\left(x_{i}\right)=x_{i}$ for $i<k$, and

4. $\rho\left(x_{k}\right)=0$.

The elements $x_{k}$ and $B_{k, j}$ for $1 \leq j<k$ generate the free product $L\left[V_{k-1}(q)\right] \amalg L$, as by inspection there are no relations among these elements. Furthermore, this free product is the kernel of $\rho$. The cross-section follows from the definitions.

\section{Homological CAlculations for $\mathbb{R}^{m}$}

Recall that $F\left(\mathbb{R}^{m}, k\right)$ is $(m-2)$-connected and that the algebra

$$
H^{*}\left(F\left(\mathbb{R}^{m}, k\right) ; \mathbb{Z}\right)
$$

is generated by classes $A_{i, j}, k \geq i>j \geq 1$, of degree $m-1$ [4, 9]. Thus the homology suspension induces an isomorphism

$$
\sigma_{*}: H_{m-2}\left(\Omega F\left(\mathbb{R}^{m}, k\right) ; \mathbb{Z}\right) \rightarrow H_{m-1}\left(F\left(\mathbb{R}^{m}, k\right) ; \mathbb{Z}\right)
$$

for $m>2$. Define $B_{i, j}$ to be the unique class specified by $\sigma_{*}\left(B_{i, j}\right)=A_{i, j *}$, the dual basis element to $A_{i, j}$.

All commutation relations for the $B_{i, j}$ are obtained by (1) exibiting maps $\gamma$ : $S^{m-1} \times S^{m-1} \rightarrow F\left(\mathbb{R}^{m}, k\right)$, (2) looping the map $\gamma$, and (3) using the commutivity of the two fundamental cycles in $H_{*}\left(\left(\Omega S^{m-1}\right)^{2} ; \mathbb{Z}\right)$.

In this case, fix integers $s, t$, and $\ell$ such that $k \geq s>t \geq 1, k \geq \ell \geq 1$, with $\ell \notin\{s, t\}$. Define a map

$$
\gamma(s, t, \ell): S^{m-1} \times S^{m-1} \rightarrow F\left(\mathbb{R}^{m}, k\right)
$$

by the formula

$$
\gamma(s, t, \ell)(u, v)=\left(x_{1}, \ldots, x_{k}\right)
$$

with $\|u\|=\|v\|=1$ such that

1. $z_{i}=(4 i, 0,0, \ldots, 0)$ for $k \geq i \geq 1$,

2. $x_{i}=z_{i}$ if $i \neq\{s, t\}$, and

3. $x_{s}=z_{\ell}+2 v, x_{t}=z_{\ell}+u$.

Notice that $\left(x_{1}, \ldots, x_{k}\right)$ is indeed in $F\left(\mathbb{R}^{m}, k\right)$.

Next, recall that the class $A_{i, j}$ is defined by the equation

$$
A_{i, j}=\pi_{i, j}^{*}(\iota)
$$

where $\pi_{i, j}: F\left(\mathbb{R}^{m}, k\right) \rightarrow F\left(\mathbb{R}^{m}, 2\right)$ denotes projection on the $(i, j)$ coordinates and $\iota$ is a fixed fundamental cycle for $S^{m-1}$ [4, 9].

Lemma 5.1. 1. If $\{i, j\} \cap\{s, t, \ell\}$ has cardinality 0 or 1 , then

$$
\gamma(s, t, \ell)^{*}\left(A_{i, j}\right)=0 .
$$

2. If $\{i, j\} \cap\{s, t, \ell\}$ has cardinality 2 , then 
(a) for $\ell<t<s$,

$$
\gamma(s, t, \ell)^{*}\left(A_{i, j}\right)= \begin{cases}\iota \otimes 1 & \text { if } j=\ell \text { and } i=t, \\ 1 \otimes \iota & \text { if } j=\ell \text { and } i=s, \\ 1 \otimes \iota & \text { if } j=t \text { and } i=s,\end{cases}
$$

(b) for $t<\ell<s$,

$$
\gamma(s, t, \ell)^{*}\left(A_{i, j}\right)= \begin{cases}(-1)^{m} \iota \otimes 1 & \text { if } j=t \text { and } i=\ell, \\ 1 \otimes \iota & \text { if } j=t \text { and } i=s, \\ 1 \otimes \iota & \text { if } j=\ell \text { and } i=s,\end{cases}
$$

(c) for $t<s<\ell$,

$$
\gamma(s, t, \ell)^{*}\left(A_{i, j}\right)= \begin{cases}(-1)^{m} \iota \otimes 1 & \text { if } j=t \text { and } i=\ell, \\ 1 \otimes \iota & \text { if } j=t \text { and } i=s, \\ (-1)^{m} 1 \otimes \iota & \text { if } j=s \text { and } i=\ell .\end{cases}
$$

Proof. Notice that if $\{i, j\} \cap\{s, t, \ell\}=\varnothing$, then $\pi_{i, j} \circ \gamma(s, t, \ell)$ is constant. If $\{i, j\} \cap$ $\{s, t, \ell\}$ has cardinality 1 , then all but one of the $x_{r}$ coordinates are constant. Furthermore, $\pi_{i, j} \circ \gamma(s, t, \ell)(u, v)=\left(x_{i}, x_{j}\right)$, where either

1. $x_{i}=z_{i}$ and $x_{j}=z_{\ell}+2 v$ with $i \neq \ell$, or

2. $x_{i}=z_{i}$ and $x_{j}=z_{\ell}+u$ with $i \neq \ell$, or

3. $x_{i}=z_{\ell}+2 v$ or $z_{\ell}+u$ and $x_{j}=2 j, j \neq=\ell$.

In any of these three cases, $\pi_{i, j} \circ \gamma(s, t, \ell)$ is null-homotopic.

Part (ii) is obtained by considering three cases. We give only one, as the others are similar. Thus assume that $t<s<\ell$.

Case 1: $j=t$ and $i=\ell$.

In this case $\pi_{i, j} \circ \gamma(s, t, \ell)(z, w)=\left(y_{\ell}+z, y_{\ell}\right)$. This last map is evidently homotopic to the map which sends $(z, w)$ to $\left(y_{\ell}, y_{\ell}-z\right)$, and thus $A_{i, j}$ pulls back to $(-1)^{m} \iota \otimes 1$.

Case 2: $j=t$ and $i=s$.

In this case $\pi_{i, j} \circ \gamma(s, t, \ell)(z, w)=\left(y_{\ell}+z, y_{\ell}+2 w\right)$. By shrinking $z$ to 0 , this last map is homotopic to the map which sends $(z, w)$ to $\left(y_{\ell}, y_{\ell}+2 w\right)$. Hence $A_{i, j}$ pulls back to $1 \otimes \iota$.

Case 3: $j=s$ and $i=\ell$.

In this case $\pi_{i, j} \circ \gamma(s, t, \ell)(z, \omega)=\left(y_{\ell}+2 w, y_{\ell}\right)$, which is homotopic to the map that sends $(z, w)$ to $\left(y_{\ell}, y_{\ell}-w\right)$. Hence $A_{i, j}$ pulls back to $(-1)^{m} 1 \otimes \iota$.

Direct dualization of Lemma 5.1 gives the next result, where $A_{i, j *}$ denotes the dual basis element for the basis given by the $A_{i, j}$.

Lemma 5.2. 1. If $\ell<t<s$, then

$$
\begin{aligned}
& \gamma(s, t, \ell)_{*}(i \otimes 1)=A_{t, \ell *}, \text { and } \\
& \gamma(s, t, \ell)_{*}(1 \otimes i)=A_{s, \ell *}+A_{s, t *} .
\end{aligned}
$$

2. If $t<\ell<s$, then

$$
\begin{aligned}
& \gamma(s, t, \ell)_{*}(i \otimes 1)=(-1)^{m} A_{\ell, t *}, \text { and } \\
& \gamma(s, t, \ell)_{*}(1 \otimes i)=A_{s, \ell *}+A_{s, \ell *} .
\end{aligned}
$$


3. If $t<\ell<s$, then

$$
\begin{aligned}
& \gamma(s, t, \ell)_{*}(i \otimes 1)=(-1)^{m} A_{\ell, t *}, \text { and } \\
& \gamma(s, t, \ell)_{*}(1 \otimes i)=A_{s, t *}+(-1)^{m} A_{\ell, s *} .
\end{aligned}
$$

Next consider the two "axial" inclusions $S^{m-1} \rightarrow S^{m-1} \times S^{m-1}$. Passage to adjoints gives two classes $x_{i}$ in $H_{m-2}\left(\Omega\left(S^{m-1}\right)^{2} ; \mathbb{Z}\right)$ such that $H_{*}\left(\Omega\left(S^{m-1}\right)^{2} ; \mathbb{Z}\right)$ is isomorphic to $T\left[x_{1}\right] \otimes T\left[x_{2}\right]$ as an algebra, where

$$
\left[x_{1}, x_{2}\right]=x_{1} x_{2}-(-1)^{m} x_{2} x_{1}=0 .
$$

Thus $\Omega \gamma(s, t, \ell)_{*}=\left[x_{1}, x_{2}\right]=0$ by naturality. The infinitesimal braid relations arise by applying this formula to Lemma 4.2 .

Corollary 5.3. If $m \geq 3$, then the following relations hold in

$$
H_{*}\left(\Omega F\left(\mathbb{R}^{m}, k\right) ; \mathbb{Z}\right):
$$

1. $\left[B_{i, j}, B_{i, t}+(-1)^{m} B_{t, j}\right]=0$ if $1 \leq j<t<i \leq k$.

2. $\left[B_{t, j}, B_{i, j}+B_{i, t}\right]=0$ if $1 \leq j<t<i \leq k$.

Proof. By Lemma 5.2 and the definition that $B_{i, j}$ is the unique element such that $\sigma_{*} B_{i, j}=A_{i, j *}$, the following holds:

(i) If $\ell<t<s$, then

$$
\begin{aligned}
& \Omega \gamma(s, t, \ell)_{*}\left(x_{1} \otimes 1\right)=B_{t, \ell}, \text { and } \\
& \Omega \gamma(s, t, \ell)_{*}\left(1 \otimes x_{2}\right)=B_{s, \ell}+B_{s, t} .
\end{aligned}
$$

1. If $t<\ell<s$, then

$$
\begin{aligned}
& \Omega \gamma(s, t, \ell)_{*}\left(x_{1} \otimes 1\right)=(-1)^{m} B_{\ell, t}, \text { and } \\
& \Omega \gamma(s, t, \ell)_{*}\left(1 \otimes x_{2}\right)=B_{s, t}+B_{s, \ell} .
\end{aligned}
$$

2. If $t<s<\ell$, then

$$
\begin{aligned}
& \Omega \gamma(s, t, \ell)_{*}\left(x_{1} \otimes 1\right)=(-1)^{m} B_{\ell, t}, \text { and } \\
& \Omega \gamma(s, t, \ell)_{*}\left(1 \otimes x_{2}\right)=B_{s, t}+(-1)^{m} B_{\ell, s} .
\end{aligned}
$$

Thus part (i) gives $\left[B_{t, \ell}, B_{s, \ell}+B_{s, t}\right]=0$ for $\ell<t<s$. This is a restatement of equation (2). In addition, part (iii) gives

$$
\left[B_{\ell, t},(-1)^{m} B_{s, t}+B_{\ell, s}\right]=0 \quad \text { if } t<s<\ell .
$$

This is a restatement of equation (2). The lemma follows.

Proposition 5.4. If $m \geq 3$ and $\{i, j\} \cap\{s, t\}=\varnothing$, then

$$
\left[B_{i, j}, B_{s, t}\right]=0
$$

in $H_{*}\left(\Omega F\left(\mathbb{R}^{m}, k\right) ; \mathbb{Z}\right)$.

Proof. The proof here is similar to that of 5.3. If $\{i, j\} \cap\{s, t\}=\varnothing$, then define

$$
\theta: S^{m-1} \times S^{m-1} \rightarrow F\left(\mathbb{R}^{m}, k\right)
$$

by the formula

$$
\theta(u, v)=\left(x_{1}, \ldots, x_{v}\right),
$$


where the $x_{i}$ are defined by (1) $x_{k}=z_{k}$ for $k \notin\{i, j, s, t\},(2) \quad x_{j}=z_{j}, x_{i}=z_{j}+u$, (3) $x_{s}=z_{s}$, and (4) $x_{t}=z_{s}+v$. Then

$$
\begin{aligned}
& \theta_{*}(\iota \otimes 1)=A_{i, j_{*}}, \\
& \theta_{*}(1 \otimes \iota)=A_{s, t *} .
\end{aligned}
$$

Furthermore, $(\Omega \theta)_{*}\left(x_{1} \otimes 1\right)=B_{i, j}$ and $(\Omega \theta)_{*}\left(1 \otimes x_{2}\right)=B_{s, t}$. Since $\left[x_{1}, x_{2}\right]=0$, it follows that $\left[B_{i, j}, B_{s, t}\right]=0$ by naturality.

\section{EnVELOPING ALGEBRAS AND THE PROOF OF 2.3}

Consider the fibration

$$
\Omega(\pi): \Omega F\left(\mathbb{R}^{m}, k\right) \rightarrow \Omega F\left(\mathbb{R}^{m}, k-1\right)
$$

with the fibre $\Omega\left(\mathbb{R}^{m}-Q_{k-1}\right)$, where $\pi$ is projection on the first $k-1$ coordinates.

By the definition of $A_{i, j *}$ and $B_{i, j}$,

$$
\Omega(\pi)_{*} B_{i, j}= \begin{cases}B_{i, j} & \text { if } i \leq k-1 \\ 0 & \text { if } i=k\end{cases}
$$

Furthermore, $H_{*}\left(\Omega F\left(\mathbb{R}^{m}, k\right) ; \mathbb{Z}\right)$ is an associative algebra for which the infinitesimal braid relations are satisfied for the $B_{i, j}, i \leq j<i \leq k$ (by the results in Section 4).

Thus there is a morphism of short exact sequences of algebras

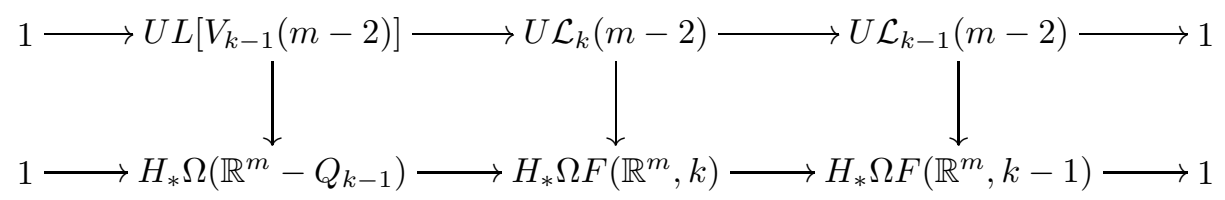

by the collapsing of the Serre spectral sequence for $\Omega(\pi)$ and by Corollary 4.2. The natural map on the level of universal enveloping algebras given by $U \mathcal{L}_{k-1}(m-2) \rightarrow$ $H_{*} \Omega F\left(\mathbb{R}^{m}, k-1\right)$ may be assumed to be an isomorphism starting with the case $k-1=2$. The map $U L\left[V_{k-1}(m-2)\right] \rightarrow H_{*} \Omega\left(\mathbb{R}^{m}-Q_{k-1}\right)$ is also an isomorphism by construction. This last statement is the conclusion of Theorem 2.3.

\section{General twistings; Proof of Theorem 2.4}

If $M$ is a $p$-manifold of dimension at least 3 , a choice of homotopy equivalence

$$
(\Omega M)^{k} \times \Omega F\left(\mathbb{R}^{m}, k\right) \times \prod_{i=1}^{k-1} \Omega \Sigma\left[(\Omega M) \wedge \Omega\left(\bigvee_{i} S^{m-1}\right)\right] \rightarrow \Omega F(M, k)
$$

was given in the proof of Theorem 2.1. This map is a product of the loop maps

1. $\Omega\left(s_{i}\right): \Omega M \rightarrow \Omega F(M, k), 1 \leq i \leq k$,

2. $\Omega e: \Omega F\left(\mathbb{R}^{m}, k\right) \rightarrow \Omega F(M, k)$, and

3. $\Omega \gamma_{i}: \Omega \Sigma\left[(\Omega M) \wedge \Omega\left(\bigvee_{i} S^{m-1}\right)\right] \rightarrow \Omega F(M, k), 1 \leq i \leq k$ (with $\gamma_{1}$ being null).

Thus if $E_{*}$ is a homology theory which satisfies the strong form of the Künneth theorem for $(\Omega M)^{\ell}$, then $E_{*} \Omega F(M, k)$ is generated as an algebra by the images of

1. $E_{*}(\Omega M)^{k}$,

2. $E_{*} \Omega F\left(\mathbb{R}^{m}, k\right)$, and

3. $E_{*} \Omega \Sigma\left[(\Omega M) \wedge \Omega\left(\bigvee_{i} S^{m-1}\right)\right], 2 \leq i \leq k$. 
Assume that $E_{*}(\Omega M)$ is given. By Theorem 2.3, $E_{*} \Omega F\left(\mathbb{R}^{m}, k\right)$ is known. Furthermore, $E_{*} \Omega \Sigma\left[(\Omega M) \wedge \Omega\left(\bigvee_{i} S^{m-1}\right)\right]$ is generated by the images of $\bar{E}_{*}\left[(\Omega M) \wedge S^{t}\right]$ for various choices of $t$ (one $S^{t}$ for each choice of basis element in $H_{*}\left(\Omega\left(\bigvee_{i} S^{m-1}\right) ; \mathbb{Z}\right.$ ) in degree $t>0)$. In addition, these generators are induced by applying $E_{*}$ to the map of spaces induced by the Samelson product

$$
(\Omega M) \wedge \Omega\left(\bigvee_{k-1} S^{m-1}\right) \rightarrow \Omega\left(M-Q_{k-1}\right) \rightarrow \Omega F(M, k) .
$$

Thus to determine $E_{*} \Omega F(M, k)$ as an algebra, it suffices to analyze the commutation relations of

1. the $B_{i, j}, k \geq i>j \geq 1$, and

2. the classes $m_{\ell}$ which are those in the image of the map in homology induced by

$$
\Omega\left(s_{\ell}\right): \Omega M \rightarrow \Omega F(M, k) .
$$

Notice that by Theorems 2.1 and 2.3, the infinitesimal braid relations in 3(i) are satisfied. The relation $\left[B_{i, j}, m_{\ell}\right]=0$ if $\ell=\notin\{i, j\}$ will be derived next, but the proof of relation 3(iii) will be postponed until section 11, as that requires some additional topology.

Consider the embedding $e: \mathbb{R}^{m} \rightarrow M$ which is used in the proof of Theorem 2.1. Since $M$ is a $p$-manifold, $M-e\left(\mathbb{R}^{m}\right)$ is homotopy equivalent to $M-\{$ point $\}$ and thus $M \vee S^{m-1}$. Thus there are choices of pointed maps

1. $\delta: M \rightarrow M-e\left(\mathbb{R}^{m}\right)$, and

2. $\mu: S^{m-1} \rightarrow M-e\left(\mathbb{R}^{m}\right)$

such that the induced map

$$
M \vee S^{m-1} \rightarrow M-e\left(\mathbb{R}^{m}\right)
$$

is a homotopy equivalence.

Next, let $X_{m}$ be the subspace of $\mathbb{R}^{m}$ given by the points of norm less than 1 . Of course $X_{m}$ is homeomorphic to $\mathbb{R}^{m}$. Choose points $q_{1}^{\prime}, \ldots, q_{k}^{\prime}$ in $\mathbb{R}^{m}$ of norm at least 2 (where one choice is given by $q_{i}^{\prime}=(1+i, 0, \ldots, 0)$ ). Let $q_{i}=e\left(q_{i}^{\prime}\right)$. These constructions are used to give maps

$$
\beta_{\ell, i, j}: F\left(X_{m}, 2\right) \times M \rightarrow F(M, k),
$$

where $1 \leq j<i \leq k$ and $\ell \notin\{i, j\}$. Define

$$
\beta_{\ell, i, j}\left(\left(x_{1}, x_{2}\right), m\right)=\left(z_{1}, \ldots, z_{k}\right)
$$

with

1. $z_{j}=e\left(x_{1}\right)$,

2. $z_{i}=e\left(x_{2}\right)$,

3. $z_{\ell}=\delta(m)$, and

4. $z_{t}=q_{t}$ if $t \notin\{i, j, \ell\}$.

Lemma 7.1. If $1 \leq j<i \leq k$ and $\ell \notin\{i, j\}$ then the maps

$$
\beta_{\ell, i, j}: F\left(X_{m}, 2\right) \times M \rightarrow F(M, k)
$$

satisfy the following properties:

1. the $\beta_{\ell, i, j}$ restricted to $M$ are homotopic to the $s_{\ell}$, the maps used in the statement of Theorem 2.4, and 
2. $\beta_{\ell, k, j}$ restricted to $F\left(X_{m}, 2\right)$ is homotopic to the map which sends $\left(x_{1}, x_{2}\right)$ to $\left(z_{1}, \ldots, z_{k}\right)$ with $z_{j}=e\left(x_{1}\right), z_{i}=e\left(x_{2}\right)$, and $z_{t}=q_{t}$ for $t \neq i, j$.

Proof. To check (1), notice that $\beta_{\ell, i, j}$ restricted to $M$ sends a point $m$ to $\left(z_{1}, \ldots, z_{k}\right)$ with $z_{\ell}=\delta(m)$. This map is homotopic to the composite

$$
M \rightarrow M-e\left(\mathbb{R}^{m}\right) \subset M-e\left(\mathbb{R}^{m}\right) \cup\left\{q_{1}, \ldots, q_{k}\right\} \rightarrow F(M, k)
$$

given by $s_{\ell}$ as defined before Theorem 2.4 .

To check (2), notice that $\Omega\left(\beta_{\ell, i, j}\right)$ restricted to $F\left(X_{m}, 2\right)$ is homotopic to the map which sends $\left(x_{1}, x_{2}\right)$ to $\left(z_{1}, \ldots, z_{k}\right)$ with

1. $z_{j}=e\left(x_{1}\right)$,

2. $z_{i}=e\left(x_{2}\right)$, and

3. $z_{t}=q_{t}$ for $t \neq i, j$.

This suffices.

To finish this section, the next statement will be proven.

Lemma 7.2. With the hypotheses in Theorem 2.4,

$$
\left[B_{i, j}, m_{\ell}\right]=0
$$

if $1 \leq j<i \leq k$ and $\ell \notin\{i, j\}$.

Proof. Consider

$$
\beta_{\ell, i, j}: F\left(X_{m}, 2\right) \times M \rightarrow F(M, k)
$$

and the looping of this map

$$
\Omega\left(\beta_{\ell, i, j}\right): \Omega F\left(X_{m}, 2\right) \times \Omega M \rightarrow \Omega F(M, k) .
$$

Thus the elements in the image of $E_{*} \Omega F\left(X_{m}, 2\right)$ and $E_{*} \Omega M$ commute. By Lemma 7.1, the image of $E_{*} \Omega M$ is precisely those elements $m_{\ell}$. By definition, $B_{i, j}$ is in the image of the fundamental cycle in $E_{m-2} \Omega F\left(X_{m}, 2\right)$. The lemma follows.

Theorem 2.4 follows at once.

\section{Commutativity Relations; Proofs of Theorems 2.6 and 2.7}

Let $M$ be a manifold which satisfies the hypotheses of Theorem 2.6. Thus $M$ is a $p$-manifold, and is "braidable". Recall that if $M$ is a $p$-manifold, there are maps $s_{i}: M \rightarrow F(M, k)$ defined after Theorem 2.3. Thus there are induced maps $\Omega\left(s_{i}\right): \Omega M \rightarrow \Omega F(M, k)$. Classes $m_{i}$ in the reduced homology $\bar{H}_{*}(\Omega F(M, k) ; \mathbb{F})$ are defined by the equation

$$
\Omega\left(s_{i}\right)_{*}(m)=m_{i}
$$

for a homology class $m$ in $\bar{H}_{*}(\Omega M ; \mathbb{F})$. The conclusion of Theorem 2.6 to be proven is that $\left[m_{i}, m_{j}^{\prime}\right]=0$ in $H_{*}(\Omega F(M, k) ; \mathbb{F})$ for any classes $m, m^{\prime}$ with $i>j$.

By Definition 2.5 of a "braidable" manifold, there exists a non-empty space $A$ together with embeddings

$$
e_{i}: A \rightarrow M
$$

with $e_{1}(A) \cap e_{2}(A)=\varnothing$. Furthermore, since $M$ is a $p$-manifold, it can be assumed that there are embeddings

$$
e_{i}^{\prime}: A \rightarrow M
$$


and

$$
e: \mathbb{R}^{m} \rightarrow M
$$

such that

1. $e_{1}^{\prime}(A) \cap e_{2}^{\prime}(A)=\varnothing$,

2. $e_{i}^{\prime}(A) \cap e\left(\mathbb{R}^{m}\right)=\varnothing$, and

3. $e_{i}^{\prime}$ isotopic to $e_{i}$.

The last assertion follows by regarding $M-e_{2}(A) \subset M-\{$ point $\} \subset M^{\prime}-\{$ point $\}$ with an embedding $M \subset M^{\prime}$ which is also a homotopy equivalence.

Fix integers $i$ and $j$ as above with $j<i$. Define

$$
\psi: A \times A \rightarrow F(M, k)
$$

by the formula

$$
\psi\left(a_{1}, a_{2}\right)=\left(z_{1}, \ldots, z_{k}\right)
$$

where

1. $z_{j}=e_{1}\left(a_{1}\right)$,

2. $z_{i}=e_{2}\left(a_{2}\right)$, and

3. $z_{t}=e\left(q_{t}\right)$ for all $t \neq i, j$.

Notice that $\psi$ restricted to the first copy of $A$ is homotopic to the composite

$$
A \stackrel{e_{1}}{\longrightarrow} M \stackrel{s_{j}}{\longrightarrow} F(M, k) .
$$

Furthermore, $\psi$ restricted to the second copy of $A$ is homotopic to the composite

$$
A \stackrel{e_{2}}{\longrightarrow} M \stackrel{s_{i}}{\longrightarrow} F(M, k) .
$$

Consider the loop map

$$
\Omega(\psi): \Omega(A \times A) \rightarrow \Omega(F(M, k)) .
$$

Thus, given classes $a_{i}$ in $\bar{H}_{*}(\Omega(A) ; \mathbb{F})$, the classes $a_{1} \otimes 1$, and $1 \otimes a_{2}$ commute in $H_{*}(\Omega(A \times A) ; \mathbb{F})$, and so $\Omega(\psi)_{*}\left[a_{1} \otimes 1,1 \otimes a_{2}\right]=0$.

By definition, the maps $\Omega\left(e_{i}\right): \Omega A \rightarrow \Omega M$ induce surjections in homology (with $R$-coefficients). It follows that the images of $\Omega\left(e_{1}\right)_{*}$ commute with the images of $\Omega\left(e_{2}\right)_{*}$ and thus $\left[m_{i}, m_{j}^{\prime}\right]=0$. Theorem 2.6 follows.

To prove Theorem 2.7, we show that certain choices of manifolds are "braidable". The first case is given by (1) $M=N \times \mathbb{R}^{1}$, for which it must be checked that $M$ is "braidable" with $A=N$. Define

$$
e_{i}: N \rightarrow N \times \mathbb{R}^{1}
$$

by $e_{i}(x)=(x, i)$ for $i=1,2$. This suffices.

To check case (2), assume that the dimension of $M$ is greater than twice the homological dimension of $M$. Thus there exist maps $e_{i}: A \rightarrow M, i=1,2$, which (1) are embeddings, and (2) induce homology isomorphisms. With the hypotheses above, $A$ may be taken to be the spine of $M$ with isotopies inducing $e_{1}(A) \cap e_{2}(A)=$ $\varnothing$.

To check case (3), consider $M=M_{1} \times M_{2} \times M_{3}$, an $m$-dimensional manifold with $e: D^{m} \rightarrow M$ an embedded $m$-disk, and each $M_{i}$ of dimension at least 1 . Fix two distinct points $a_{i}, b_{i}$ in $M_{i}$ (with $a_{i} \neq b_{i}$ ).

Define $A_{1}$ to be equal to the subspace of $M$ given by $\left(m_{1}, m_{2}, m_{3}\right)$ with at least 2 coordinates given by $a_{i}, 1 \leq i \leq 3$. Define $A_{2}$ to be equal to the subspace of $M$ given by $\left(m_{1}, m_{2}, m_{3}\right)$ with at least 2 coordinates given by $b_{i}, 1 \leq i \leq 3$. Notice 
that $A_{1}$ and $A_{2}$ are disjoint subsets of $M$. Since each $M_{i}$ is path-connected, the evident analogue of a Dehn twist gives isotopies $h_{i}$ of $M_{i}$ which carry $a_{i}$ to $b_{i}$ with

$$
h_{i}:[0,1] \times M_{i} \rightarrow M_{i},
$$

where

1. $h_{i}\left(0, m_{i}\right)=m_{i}$,

2. $h_{i}(t,-)$ is an embedding, and

3. $h_{0}\left(1, a_{i}\right)=b_{i}$.

Thus there is a map

$$
h: A_{1} \rightarrow A_{2}
$$

given by $h\left(m_{1}, m_{2}, m_{3}\right)=\left(h_{1}\left(1, m_{1}\right), h_{2}\left(1, m_{2}\right), h_{3}\left(1, m_{3}\right)\right)$. Define $A=A_{1}$. The natural embedding $e_{2}: A_{2} \rightarrow M$ precomposed with the embedding $h$ gives an embedding $e=e_{2} \circ h$ which is isotopic to the natural one $e_{1}: A_{1} \rightarrow M$. Thus $e_{1}$ and $e_{2}$ are isotopic with $e_{1}(A) \cap e_{2}(A)=\varnothing$.

Since $A_{i}$ is homotopy equivalent to $M_{1} \vee M_{2} \vee M_{3}$, the induced maps $\Omega\left(e_{i}\right)$ induce surjections in homology (as $\Omega(A \vee B$ ) is homotopy equivalent to $\Omega A \times \Omega B \times$ $\Omega \Sigma[(\Omega A) \wedge(\Omega B)]$ for path-connected pointed spaces $A$ and $B)$. Theorem 2.7 follows.

\section{Proof of Theorem 2.8}

The purpose of this section is to prove Theorem 2.8. The content of this theorem is that there exist classes $m$ and $m^{\prime}$ in $H_{*}(\Omega M ; \mathbb{F})$ with

$$
\left[m_{i}, m_{j}^{\prime}\right] \neq 0
$$

in $H_{*}(\Omega F(M, k) ; \mathbb{F})$ for $k \geq i>j \geq 1$ in case $M$ is one of the following manifolds:

1. $S^{p} \times S^{q}-Q_{t}, t \geq 0, p, q \geq 2$,

2. $S U(3)-Q_{t}, t \geq 0$,

3. $S p(2)-Q_{t}, t \geq 0$,

4. $V_{n, 2}-Q_{t}, t \geq 0$, with $\mathbb{F}=\mathbb{F}_{2}$ in the cases where $n$ is odd, and where $V_{n, 2}$ denotes the Stiefel manifold of ordered orthonormal 2-frames in $\mathbb{R}^{n}$ with $n>3$, or

5. $M$ is a connected sum $A \sharp B$ with $B$ as in (1)-(4).

Throughout this section $M$ will be assumed to denote one of the manifolds in (1)-(5) above. In addition, the proof given below is presented in two parts.

1. The first part is the construction of a "universal example" for such types of commutators in homology via a two-stage Postnikov tower.

2. The second part is the construction of a map to the universal example satisfying certain properties listed below.

Thus if $M$ is closed, there exist classes $u$ and $v$ of degree $p>0$ and $q>0$ respectively in the cohomology ring of $M$ with the cup product $u \cup v$ equal to the fundamental class $[M]$. Of course, if $M$ is given by the connected sum of a manifold $A$ with the Stiefel manifold $V_{n, 2}$ for $n$ odd, then cohomology is assumed to be taken with $\mathbb{F}=\mathbb{F}_{2}$ coefficients.

Consider the inclusion $i_{M}: F(M, 2) \rightarrow M^{2}$. Let

1. $C\left(i_{M}\right)$ denote the cofibre of $i_{M}$,

2. $Y_{M}$ denote the homotopy fibre of $i_{M}$, and

3. $\tau_{M}$ denote the tangent bundle of $M$.

Part (1) of the next lemma is Lemma 11.5 in [26]. 
Lemma 9.1. $\quad$ 1. If $M$ is a Riemannian manifold, then the cofibre $C\left(i_{M}\right)$ is the Thom complex $T\left(\tau_{M}\right)$ of the tangent bundle $\tau_{M}$ of $M$.

2. The homotopy theoretic fibre $Y_{M}$ is homotopy equivalent to the homotopy theoretic fibre of the inclusion $g: M-\{$ point $\} \rightarrow M$. Thus if $M$ is a $p$ manifold of dimension greater than 2 , then $g$ admits a section up to homotopy, and $\Omega Y_{M}$ is homotopy equivalent to

$$
\Omega S^{m-1} \times \Omega \Sigma\left[(\Omega M) \wedge \Omega\left(S^{m-1}\right)\right]
$$

Proof. Since $M$ is a Riemannian manifold, $F(M, 2)$ is homotopy equivalent to the complement of a regular neighborhood $V$ of the diagonal $\Delta M$ in $M^{2}$. Collapsing $M^{2}-V$ in $M^{2}$ to a point gives the Thom complex for the tangent bundle of $M$. Part (1) follows.

To obtain part (2), consider the morphism of fibrations

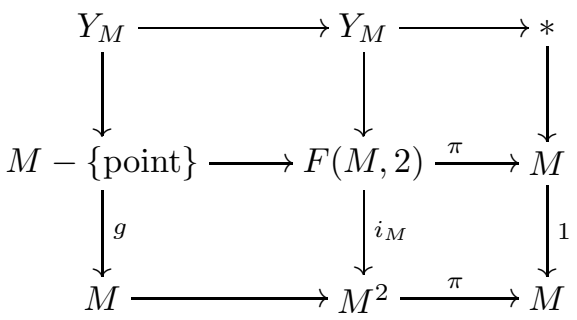

where $\pi$ is first coordinate projection. Thus $Y_{M}$ is the homotopy theoretic fibre of the inclusion $g: M-\{$ point $\} \rightarrow M$. Regard the map $g$ as the projection map from the total space of a fibration to the base.

If, in addition, $M$ is a $p$-manifold, there is a section up to homotopy for the map $g$ because $M-\{$ point $\}$ is homotopy equivalent to $M \vee S^{m-1}$ with the inclusion induced by pinching $S^{m-1}$ to a point. Thus, after looping, $\Omega(M-\{$ point $\})$ is homotopy equivalent to

$$
\Omega M \times \Omega S^{m-1} \times \Omega \Sigma\left[\Omega(M) \wedge \Omega\left(S^{m-1}\right)\right] .
$$

Hence $\Omega\left(Y_{m}\right)$ is homotopy equivalent to $\Omega S^{m-1} \times \Omega \Sigma\left[\Omega(M) \wedge \Omega\left(S^{m-1}\right)\right]$, as the map $\Omega g: \Omega(M-$ p point $\}) \rightarrow \Omega M$ is null-homotopic when restricted to $\Omega S^{m-1} \times$ $\Omega \Sigma\left[\Omega(M) \wedge \Omega\left(S^{m-1}\right)\right]$. The lemma follows.

Returning to the case for which $M$ is closed and orientable, consider the cofibration

$$
F(M, 2) \stackrel{i_{M}}{\longrightarrow} M^{2} \stackrel{j}{\longrightarrow} T\left(\tau_{M}\right)
$$

with

$$
j^{*}(U)=[M] \otimes 1+1 \otimes[M]+\Sigma x \otimes x^{*},
$$

where $U$ is the Thom class, with cup-product $x \cup x^{*}=[M]$, the fundamental cycle [26]. If $M$ is not closed, a similar formula holds where $[M]$ is set equal to zero.

Thus, if $M$ is closed and orientable, there exist classes $u$ and $v$ with $u \cup v=[M]$. Thus

$$
j^{*}(U)=[M] \otimes 1+1 \otimes[M]+u \otimes v+(-1)^{|u||v|} v \otimes u+\text { "others" }
$$

where

1. "others" consist of $\sum_{x} x \otimes x^{*}$, and

2. the elements $u \otimes v, v \otimes u$, and $x \otimes x^{*}$ are linearly independent. 
Similar formulas hold in case $M$ is closed, not necessarily orientable, and coefficients are taken in $\mathbb{F}_{2}$.

It is convenient to index an Eilenberg-Mac Lane spaces by the "name" of a cohomology class in the proofs given below. For this we need some more notation.

Let $\mathbb{F}$ denote a field with either $\mathbb{F}=\mathbb{F}_{p}$ for a prime $p$, or $\mathbb{F}=\mathbb{Q}$. Let $y$ denote a cohomology class in $H^{q}(X ; \mathbb{F})$. The notation used below is described as follows.

1. Let $K(y)$ denote the Eilenberg-Mac Lane space $K(\mathbb{F}, q)$ for a fixed non-trivial cohomology class $y$ in $H^{q}(X ; \mathbb{F})$.

2. Let $f$ denote a map $f: X \rightarrow K(y)$ with

$$
f^{*}\left(\iota_{q}\right)=y
$$

where $\iota_{q}$ denotes a choice of fundamental class for $K(\mathbb{F}, q)$.

Consider the cohomology classes $u \otimes 1,1 \otimes u, v \otimes 1,1 \otimes v,[M] \otimes 1,1 \otimes[M], x \otimes 1$, and $1 \otimes x^{*}$ in $H^{*}\left(M^{2} ; \mathbb{F}\right)$. Let $K$ and $K^{\prime}$ denote the product of Eilenberg-Mac Lane spaces determined by these cohomology classes with

$$
K=[K(u \otimes 1) \times K(1 \otimes v)] \times[K(v \otimes 1) \times K(1 \otimes u)] \times\left[\prod_{x} K(x \otimes 1) \times K\left(1 \otimes x^{*}\right)\right]
$$

and

$$
K^{\prime}=K \times K([M] \otimes 1) \times K(1 \otimes[M]) .
$$

1. Define a map

$$
\rho: K \rightarrow K^{\prime}
$$

given by the inclusion $K \times$ \{point $\}$ in $K^{\prime}$.

2. Define a map

$$
\Phi^{\prime}:(M)^{2} \rightarrow K^{\prime}
$$

given by the value of $j^{*}(U)$ in $H^{*}\left((M)^{2}\right)$.

3. Since $[M]$ pulls back to 0 in $H^{*}\left(M-Q_{i}, \mathbb{F}\right)$ for all $i>0$, there is a lift of $\Phi^{\prime}$ given by

$$
\Phi:\left(M-Q_{1}\right)^{2} \rightarrow K
$$

The maps

$$
\Phi:\left(M-Q_{1}\right)^{2} \rightarrow K,
$$

and

$$
\Phi^{\prime}:(M)^{2} \rightarrow K^{\prime}
$$

are specified by the cohomology classes given by the "names" for $j^{*}(U)$. Thus, there is a homotopy commutative diagram

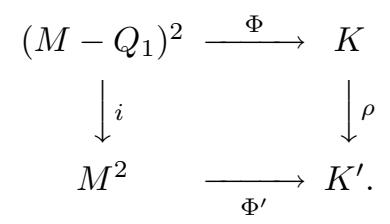


The "universal examples" $E_{\lambda}$ and $E_{\lambda^{\prime}}$ mentioned at the beginning of this section are constructed next. Define

$$
\lambda^{\prime}: K^{\prime} \rightarrow K(\mathbb{F}, m)
$$

and

$$
\lambda: K \rightarrow K(\mathbb{F}, m)
$$

by the equations

1. $\left(\lambda^{\prime}\right)^{*}\left(\iota_{m}\right)=[M] \otimes 1+1 \otimes[M]+u \otimes v+(-1)^{|u||v|} v \otimes u+\sum_{x} x \otimes x^{*}$, where $\iota_{m}$ denotes the fundamental cycle for $K(\mathbb{F}, m)$, and

2. $\lambda=\lambda^{\prime} \circ \rho$.

Define $E_{\lambda}$ and $E_{\lambda^{\prime}}$ as the homotopy theoretic fibres of $\lambda$ and $\lambda^{\prime}$ respectively, and notice that there is a map of fibrations

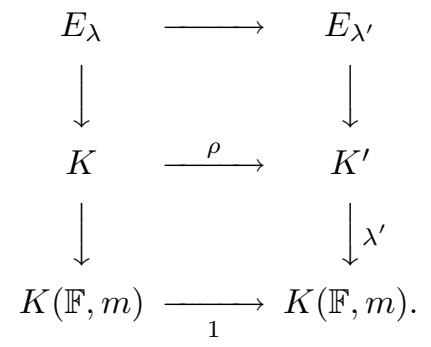

The spaces $E_{\lambda}$ and $E_{\lambda^{\prime}}$ are used below in order to obtain some multiplicative relations for the homology of the loop space of the configuration space as given in the proof of Theorem 2.8. Properties of the loop space $\Omega\left(E_{\lambda}\right)$ are given in the next two lemmas.

Lemma 9.2. As a space, $\Omega E_{\lambda}$ is homotopy equivalent to

$$
\Omega K \times K(\mathbb{F}, m-2) .
$$

Proof. Recall that $E_{\lambda}$ is defined as the homotopy theoretic fibre of the map $\lambda=$ $\lambda^{\prime} \circ \rho$, where $\rho: K \rightarrow K^{\prime}$ given by the inclusion $K \times\{1\}$ in $K^{\prime}$, and

$$
\lambda^{\prime}: K^{\prime} \rightarrow K(\mathbb{F}, m)
$$

where

$$
(\lambda)^{*}\left(\iota_{m}\right)=u \otimes v+(-1)^{|u||v|} v \otimes u+\sum_{x} x \otimes x^{*} .
$$

Thus the value of $\lambda^{*}\left(\iota_{m}\right)$ is decomposable. Since $\Omega(\lambda)$ is trivial in cohomology, the map is null-homotopic. Since a principal fibration with section is trivial, the lemma follows.

Let $\bar{u} \otimes 1,1 \otimes \bar{v}, \bar{v} \otimes 1$, and $1 \otimes \bar{u}$ denote homology classes in $H_{*}(\Omega K ; \mathbb{F})$ which are dual to the cohomology suspensions of $u \otimes 1,1 \otimes v, v \otimes 1$, and $1 \otimes u$ (respectively). These elements may be identified as elements of $H_{*}\left(\Omega E_{\lambda} ; \mathbb{F}\right)$ by Lemma 9.2. This identification is used in the next lemma.

Lemma 9.3. In $H_{*}\left(\Omega E_{\lambda} ; \mathbb{F}\right)$, the commutators

$$
[\bar{u} \otimes 1,1 \otimes \bar{v}] \text { and }[1 \otimes \bar{u}, \bar{v} \otimes 1]
$$

are equal to non-zero scalar multiples of the image of the fundamental cycle for the map $H_{m-2} \Omega K(\mathbb{F}, m-1) \rightarrow H_{m-2} \Omega E_{\lambda}$. Furthermore, the classes $\bar{u} \otimes 1$, and $1 \otimes \bar{v}$, and $[\bar{u} \otimes 1,1 \otimes \bar{v}]$ and, $[1 \otimes \bar{u}, \bar{v} \otimes 1]$ are spherical. 
Proof. There are maps

1. $u \otimes 1: S^{p} \times S^{q} \rightarrow K \times K$,

2. $1 \otimes v: S^{p} \times S^{q} \rightarrow K \times K$,

specified by the classes $u \otimes 1$ and $1 \otimes v$ in $H^{*}(K ; \mathbb{F})$. After taking products of these maps, there is an induced map

$$
b: S^{p} \times S^{q} \rightarrow K .
$$

The following diagram homotopy commutes, where $c: S^{p} \times S^{q} \rightarrow S^{p+q}$ and $\hat{b}$ induce isomorphisms on $H_{p+q}(-; \mathbb{F})$ :

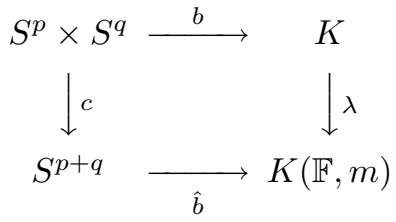

Hence there is a map of a cofibration to a fibration

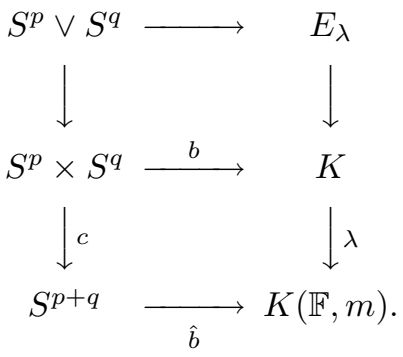

Passage to the homotopy fibre of the inclusion $S^{p} \vee S^{q} \rightarrow S^{p} \times S^{q}$ (given by $\left.\Sigma\left(\Omega S^{p}\right) \wedge\left(\Omega S^{q}\right)\right)$ together with the Serre exact sequence provides a morphism of fibrations

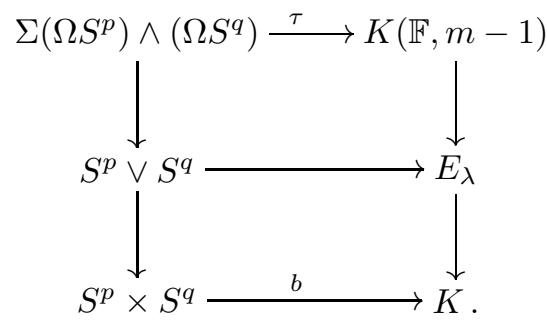

The commutator

$$
\left[\iota_{p-1}, \iota_{q-1}\right]
$$

is a non-zero multiple of a generator in $\left.H_{p+q-2}\left(\Omega \Sigma\left(\Omega S^{p}\right) \wedge\left(\Omega S^{q}\right)\right) ; \mathbb{F}\right)$. Since $\Omega(\tau)$ induces a split monomophism in $H_{p+q-2}(-; \mathbb{F})$ by Lemma 9.2 , the lemma follows by looping this fibration, and applying naturality.

Maps from configuration spaces to the space $E_{\lambda}$ are given next. The constructions of these maps are given by a comparison of the previous maps

$$
\Phi:\left(M-Q_{1}\right)^{2} \rightarrow K
$$

and

$$
\Phi^{\prime}:(M)^{2} \rightarrow K^{\prime}
$$


together with properties of the tangent bundle $\tau_{M}$ given in Lemma 9.1.

Recall that $T\left(\tau_{M}\right)$ denotes the Thom complex for the tangent bundle of $M$. By cohomological properties given in Lemma 9.1, there exist maps $\Upsilon: T\left(\tau_{M-Q_{1}}\right) \rightarrow$ $K(\mathbb{F}, m)$ and $\Upsilon^{\prime}: T\left(\tau_{M}\right) \rightarrow K(\mathbb{F}, m)$ which yield the following two homotopy commutative diagrams:

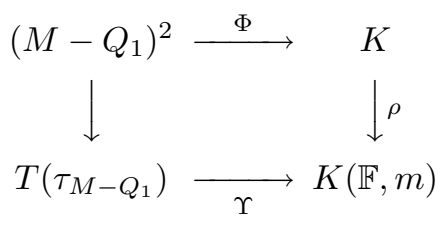

and

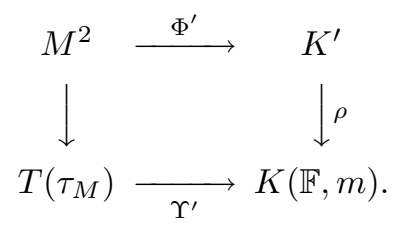

This information is used below to prove the next lemma.

Lemma 9.4. If $M$ is closed, there are morphisms of fibrations

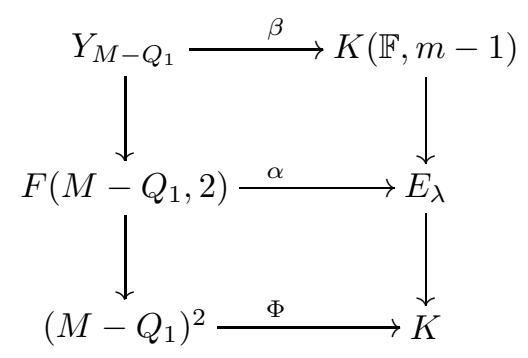

and

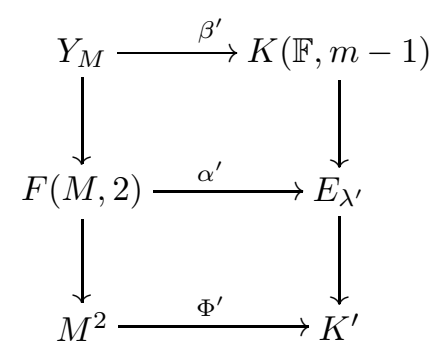

where the maps $\beta$ and $\beta^{\prime}$ are non-trivial in cohomology.

Proof. Consider the homotopy commutative diagram

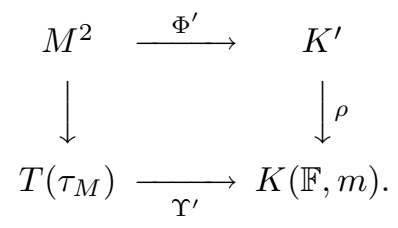


Notice that the composite $F(M, 2) \rightarrow M^{2} \rightarrow T\left(\tau_{M}\right)$ is null-homotopic. Thus there is a morphism of fibrations

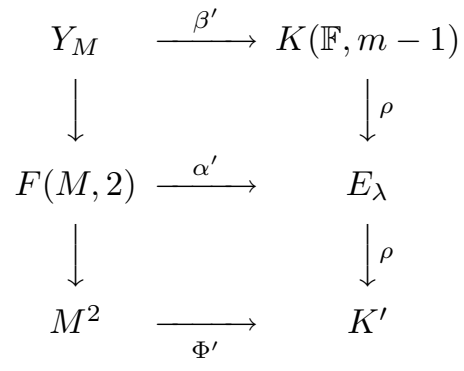

for some choice of $\alpha$ and $\beta^{\prime}$.

Notice that any choice of $\alpha^{\prime}$, and $\beta^{\prime}$ is non-trivial in cohomology by the definitions of $Y_{M}, \Phi^{\prime}$, and $\alpha^{\prime}$, by a comparison of the respective Serre spectral sequences together with Lemma 9.1. The analogous computation for which the map $\Phi^{\prime}$ is replaced by the map $\Phi$ is similar, and is omitted.

Recall that if $M$ is a $p$-manifold, and $m$ is any non-zero class in the homology of $\Omega M$, then there are sections $\sigma_{i}: M-Q_{i} \rightarrow F\left(M-Q_{i}, k-i\right)$ with non-zero classes $m_{j}$ in the homology of $\Omega F(M, k)$ given by $\left(\Omega s_{j}\right)_{*}(m)$.

Lemma 9.5. Assume that $M$ is simply-connected and that the cohomology algebra $H^{*}(M ; \mathbb{F})$ is isomorphic to an exterior algebra with two linearly independent generators. Then the loop-space homology, $H_{*}(\Omega M ; \mathbb{F})$ is isomorphic to a polynomial algebra with two generators $m$ and $m^{\prime}$. Furthermore, the elements $\left[m_{i}, m_{j}^{\prime}\right]$ as defined before the statement of Theorem 2.3 are non-zero multiples of $B_{i, j}$ in the loop-space homology of $F\left(M-Q_{i}, k\right)$ for $i>0$.

Proof. Notice that by Theorem 2.1 the space $\Omega F\left(M-Q_{i}, 2\right)$ is homotopy equivalent to $\Omega\left(M-Q_{i}\right) \times \Omega\left(M-Q_{i+1}\right)$ in case $i>0$. The natural map induced by looping the inclusion $F\left(M-Q_{i}, 2\right) \rightarrow\left(M-Q_{i}\right)^{2}$ induces an isomorphism in homology with $\mathbb{F}$ coefficients through dimension $m-3$. Furthermore, the kernel of the map

$$
H_{m-2}\left(\Omega F\left(M-Q_{i}, 2\right) ; \mathbb{F}\right) \rightarrow H_{m-2}\left(\Omega\left(M-Q_{i}\right)^{2} ; \mathbb{F}\right)
$$

for $i>0$ is one dimensional and generated by $B_{2,1}$, by Lemma 9.1.

In addition, the image of $\left[m_{1}, m_{2}^{\prime}\right]$ in $H_{m-2}\left(\Omega\left(M-Q_{i}\right)^{2} ; \mathbb{F}\right)$ is zero by inspection of the definitions. Thus the class $\left[m_{1}, m_{2}^{\prime}\right]$ is a scalar multiple of $B_{2,1}$. By Lemmas $9.2,9.3$, and 9.4, the image of $\left[m_{1}, m_{2}^{\prime}\right]$ in $H_{m-2}(\Omega K(\mathbb{F}, m-1) ; \mathbb{F})$ is non-zero. Thus the class $\left[m_{1}, m_{2}^{\prime}\right]$ is a non-zero scalar multiple of $B_{2,1}$.

Finally, notice that there is a map of $F\left(M-Q_{i}, 2\right)$ to $F\left(M-Q_{i}, k\right)$ which carries $m_{2}$ to $m_{i}$, and $m_{1}^{\prime}$ to $m_{j}^{\prime}$ if $i \neq j$. This map sends $B_{2,1}$ to $B_{i, j}$ after passage to homology of loop spaces. The lemma follows.

Proof of Theorem 2.8. This theorem is a restatement of Lemma 9.5.

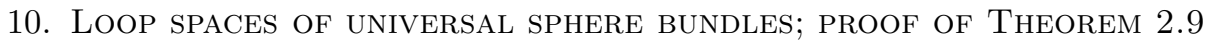

A proof of Theorem 2.9 is given in this section. Preliminary information is given first, as follows. The universal orientable $S^{n-1}$-bundle is given by

$$
S^{n-1} \rightarrow E S O(n) \times_{S O(n)} S^{n-1} \rightarrow B S O(n) .
$$


The universal $S^{n-1}$-bundle with section is

$$
S^{n-1} \rightarrow \operatorname{ESO}(n) \times_{S O(n-1)} S^{n-1} \rightarrow B S O(n-1)
$$

with $\operatorname{ESO}(n) \times_{S O(n-1)} S^{n-1}$ denoted $E_{n}$. There is a fibration $\Omega E_{n} \rightarrow S O(n-1)$ with section $s$, and so $\Omega E_{n}$ is homotopy equivalent to

$$
S O(n-1) \times \Omega S^{n-1} .
$$

Let $\iota_{n-2}$ denote a choice of generator for $H_{n-2}\left(\Omega S^{n-1} ; \mathbb{F}\right)$. If $\mathbb{F}=\mathbb{F}_{2}$, let $x_{n-2}$ denote any class in $H_{n-2}\left(S O(n-1) ; \mathbb{F}_{2}\right)$ which is dual to the cohomology suspension of the Stiefel-Whitney class $w_{n-1}$. Let $y_{n-2}$ denote any class in $H_{n-2}(S O(n-1) ; \mathbb{F})$ dual to the cohomology suspension of the Euler class in $H^{n-1}(B S O(n-1) ; \mathbb{F})$ when $n-1$ is even.

The loop space homology of $E_{n}$ is frequently non-abelian as an algebra, as will be seen below. This feature of the homology algebra will be reflected in configuration spaces in the next section. In particular, the conclusions of Theorem 2.9 are as follows:

(i): If $\mathbb{F}=\mathbb{F}_{2}$, then

$$
\left[\iota_{n-2}, x_{n-2}\right]=\iota_{n-2}^{2} \text { in } H_{*}\left(\Omega E_{n} ; \mathbb{F}_{2}\right) .
$$

(ii): If $\frac{1}{2} \in \mathbb{F}$, then

$$
\left[\iota_{n-2}, y_{n-2}\right]=\lambda\left(\iota_{n-2}\right)^{2} \text { for } \lambda \neq 0 \text { if and only if } n-1 \equiv 0(2) .
$$

Notice that the formulas in (i) and (ii) give a complete description of the loop space homology of $E_{n}$. Namely,

(iii): If $\mathbb{F}=\mathbb{F}_{2}$, then there is an extension of algebras

$$
1 \rightarrow H_{*}\left(\Omega S^{n-1} ; \mathbb{F}_{2}\right) \rightarrow H_{*}\left(\Omega E_{n} ; \mathbb{F}_{2}\right) \rightarrow H_{*}\left(S O(n-1) ; \mathbb{F}_{2}\right) \rightarrow 1,
$$

where lifts of elements for the homology algebra $H_{q}\left(S O(n-1) ; \mathbb{F}_{2}\right)$ commute with $\iota_{n-2}$ when $q<n-2$.

(iv): If $\frac{1}{2} \in \mathbb{F}$, then there is an extension of algebras when $n-1 \equiv 0(2)$,

$$
1 \rightarrow H_{*}\left(\Omega S^{n-1} ; \mathbb{F}\right) \rightarrow H_{*}\left(\Omega E_{n} ; \mathbb{F}\right) \rightarrow H_{*}(S O(n-1) ; \mathbb{F}) \rightarrow 1,
$$

where lifts of elements for $H_{q}(S O(n-1) ; \mathbb{F})$ commute with $\iota_{n-2}$ for $q<n-2$. In this case, if $n-1$ is odd, then $H_{*}\left(\Omega E_{n} ; \mathbb{F}\right)$ is abelian.

A classical calculation of 28] and [26] is recorded in the next lemma.

Lemma 10.1. The ring $H^{*}\left(E_{n} ; \mathbb{F}\right)$ is isomorphic to

$$
H^{*}(B S O(n-1) ; \mathbb{F}) \otimes H^{*}\left(S^{n-1} ; \mathbb{F}\right)
$$

as a $H^{*}(B S O(n-1) ; \mathbb{F})$-module. Let $z_{n-1}$ be any class which restricts to a generator of $H^{n-1}\left(S^{n-1} ; \mathbb{F}\right)$.

1. If $\mathbb{F}=\mathbb{F}_{2}$, then

$$
z_{n-1}^{2}=w_{n-1} z_{n-1}+v \cdot z_{n-1},
$$

where $v \in H^{n-1}\left(B S O(n-1) ; \mathbb{F}_{2}\right)$ is in the subalgebra generated by $w_{2}, \ldots$, $w_{n-2}$. 
2. If $\frac{1}{2} \in \mathbb{F}$ and $n-1$ is even, then

$$
z_{n-1}^{2}=e_{n-1} z_{n-1}+v \cdot z_{n-1},
$$

where $v \in H^{n-1}(B S O(n-1) ; \mathbb{F})$ is in the subalgebra generated by the Pontrjagin classes of degree less than $n-1$.

Consider the projection map $p: E_{n} \rightarrow B S O(n-1)=B_{n}$, and let $s: B_{n} \rightarrow E_{n}$ be a fixed choice of cross-section for $p$, with $j: S^{n-1} \rightarrow E_{n}$ the inclusion of the fibre over a point.

Lemma 10.2. There is a choice of class $z_{n-1}$ in Lemma 10.1 such that $s^{*}\left(z_{n-1}\right)=$ 0 .

Proof. By Lemma 10.1, there is a class $\bar{z}_{n-1}$ such that $j^{*}\left(\bar{z}_{n-1}\right)$ is a generator for $H^{n-1}\left(S^{n-1} ; \mathbb{F}\right)$. Define

$$
z_{n-1}=\bar{z}_{n-1}-\left(p^{*}\right)\left(s^{*}\right)\left(\bar{z}_{n-1}\right) .
$$

Thus

1. $j^{*}\left(z_{n-1}\right)=j^{*}\left(\bar{z}_{n-1}\right)$ is a generator of $H^{n-1}\left(S^{n-1} ; \mathbb{F}_{2}\right)$, and

2. $s^{*}\left(z_{n-1}\right)=s^{*}\left(\bar{z}_{n-1}\right)-s^{*} p^{*} s^{*}\left(\bar{z}_{n-1}\right)=0$.

The lemma follows.

Next, Theorem 2.9(ii) will be proven by considering the composite $\theta$ given by

$$
S^{n-1} \vee B_{n} \stackrel{j \vee s}{\longrightarrow} E_{n} \vee E_{n} \stackrel{\text { fold }}{\longrightarrow} E_{n},
$$

together with an additional map. That additional map is specified by

$$
\lambda: E_{n} \rightarrow K(\mathbb{Z}, 2 q)^{3}
$$

with $n-1=2 q$, where

(i): $\lambda^{*}(\iota \otimes 1 \otimes 1)=z_{n-1}$,

(ii): $\lambda^{*}(1 \otimes \iota \otimes 1)=e_{n-1}$, and

(iii): $\lambda^{*}(1 \otimes 1 \otimes \iota)=v$

with $z_{n-1}^{2}=e_{n-1} z_{n-1}+v \cdot z_{n-1}$ as in Lemmas 10.1 and 10.2.

Notice that

(iv): $(\lambda \theta)^{*}(\iota \otimes 1 \otimes 1)=\iota_{n-1} \otimes 1+1 \otimes s^{*}\left(z_{n-1}\right)=\iota_{n-1} \otimes 1$ by 9.2 ,

$(\mathbf{v}):(\lambda \theta)^{*}(1 \otimes i \otimes 1)=j^{*}\left(e_{n-1}\right)=+s^{*}\left(e_{n-1}\right)=1 \otimes s^{*}\left(e_{n-1}\right)$, as $j^{*}\left(e_{n-1}\right)=0$, and

$(\mathbf{v i}):(\lambda \theta)^{*}(1 \otimes 1 \otimes \iota)=j^{*}(\gamma)+s^{*}(v)=1 \otimes s^{*}(v)$.

Thus the diagram

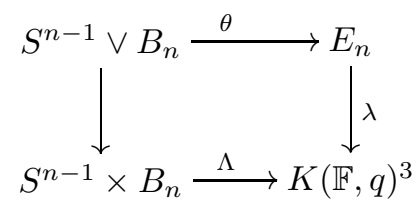

homotopy commutes, where $\Lambda$ is defined via equations (iv), (v), and (vi).

Next consider the 2-stage system given by

$$
\mu: K(\mathbb{F}, q)^{3} \rightarrow K(\mathbb{F}, 2 q)
$$


defined by

$$
\mu^{*}\left(\iota_{2 q}\right)=\left(\iota_{q} \otimes 1 \otimes 1\right)^{2}-\left(\iota_{q} \otimes \iota_{q} \otimes 1\right)-\left(\iota_{q} \otimes 1 \otimes \iota_{q}\right)
$$

with homotopy theoretic fibre denoted by $X_{q}$.

By Lemma 10.1, $(\lambda \mu)^{*}\left(\iota_{2 q}\right)$ is zero, and so there exists a choice of lift of $\lambda$ to $\tau: E_{n} \rightarrow X_{q}$ together with an induced morphism of fibration sequences where $F_{\lambda}$ denotes the homotopy theoretic fibre of $\lambda$ :

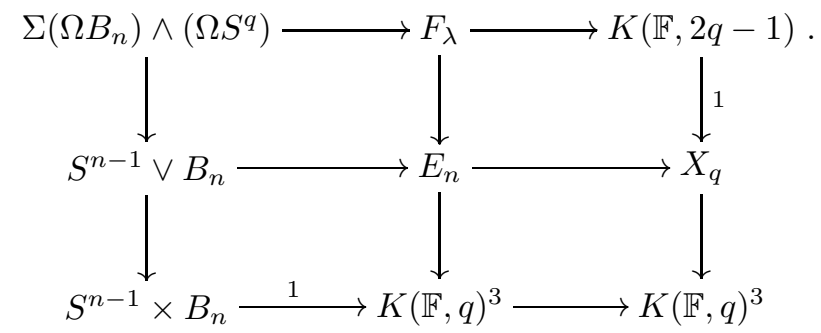

The maps in the top row are degree 1 by the Serre exact sequence. Looping the composite $\tau \circ \theta$ and appealing to naturality gives the following two lemmas.

Lemma 10.3. If $\mathbb{F}=\mathbb{F}_{2}$, then

(i): $\Omega(\tau \circ \theta)_{*}\left[\iota_{n-2} \otimes 1,1 \otimes x_{n-2}\right]=\iota_{2 q-2}$ (with $\left.2 n-4=2 q-2\right)$ in the loop space homology of $S^{n-1} \vee B$,

(ii): $\left[\iota_{n-2}, x_{n-2}\right]$ has non-trivial image in the loop space homology of $X_{q}$, and

(iii): $\left[\iota_{n-2}, x_{n-2}\right]=\iota_{n-2}^{2}$ in the loop space of homology of $E_{n}$.

Lemma 10.4. If $\frac{1}{2} \in \mathbb{F}$ and $n-1 \equiv 0(2)$, then

(i): $\Omega(\tau \circ \theta)_{*}\left[\iota_{n-2} \otimes 1,1 \otimes x_{n-2}\right]=\iota_{2 q-2}$ in the loop space homology of $S^{n-1} \vee B$ (with $2 n-4=2 q-2$ ),

(ii): $\left[\iota_{n-2}, x_{n-2}\right]$ has non-trivial image in the loop space homology of $X_{q}$, and

(iii): $\left[\iota_{n-2}, x_{n-2}\right]=\left[\iota_{n-2}, \iota_{n-2}\right]$ in the loop space homology of $E_{n}$.

Notice that Theorem 2.9 follows at once.

\section{LOOP SPACES OF TANGENT BUNDLES; COMMUTATIVITY RELATIONS IN THEOREM 2.10}

Consider the unit sphere bundle in the tangent bundle $\tau_{M, 0}$ for $M$. Thus there is a fibre bundle

$$
S^{m-1} \rightarrow \tau_{M, 0} \rightarrow M
$$

If $M$ is a $p$-manifold, there is a map described below given by

$$
\tau_{M, 0} \rightarrow F(M, K) \text {. }
$$

This map satisfies the property that the classes $B_{i, j}$ as well as the elements $m_{i}$ (which are primitive) are in the image of the induced map on the level of loop space homology. These maps will be given by compositions of maps

$$
\tau_{M, 0} \rightarrow F(M, 2), \quad \text { and } \quad F(M, 2) \rightarrow F(M, k) .
$$

The constructions below imply that the loop space homology of $\tau_{M, 0}$ will be "universal" for the relation determined by $\left[B_{i, j}, m_{i}+m_{j}\right]$ as in Theorem 2.9. Thus the first step required here is to determine the algebra obtained from the loop space homology of $\tau_{M, 0}$, for which the calculations from section 10 will be used. 
Since $M$ is assumed to be a $p$-manifold, there is a map of bundles

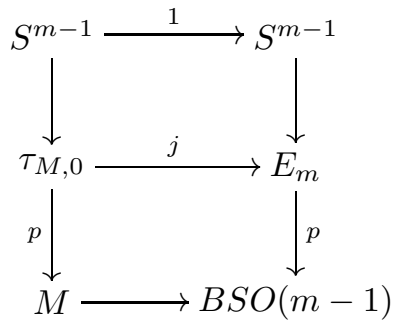

together with the induced map obtained by looping this diagram. Since right-hand $p$ admits a section $\sigma$, the induced map $S O(m-1) \rightarrow S^{m-1}$ is null. Thus the induced map $\Omega M \rightarrow S^{m-1}$ is null-homotopic, and there is a cross-section for the fibration $\Omega S^{m-1} \rightarrow \Omega \tau_{M, 0} \rightarrow \Omega M$. Since a principal fibration with a section is trivial, there is a homotopy equivalence

$$
\Omega M \times \Omega S^{m-1} \rightarrow \Omega \tau_{M, 0} .
$$

This product decompostion is used to prove the next result, which gives Theorem 2.8 .

Theorem 11.1. 1. Assume that $m-1 \equiv 1(2)$.

(a) If $\frac{1}{2} \in \mathbb{F}$, then $H_{*}\left(\Omega S^{m-1} ; \mathbb{F}\right)$ is central in $H_{*}\left(\Omega \tau_{M, 0} ; \mathbb{F}\right)$.

(b) If $\mathbb{F}=\mathbb{F}_{2}$, then $H_{*}\left(\Omega S^{m-1} ; \mathbb{F}\right)$ is central in $H_{*}\left(\Omega \tau_{M, 0} ; \mathbb{F}\right)$ if and only if $w_{m-1}\left(\tau_{M, 0}\right)=0$.

2. Assume that $m-1 \equiv 0(2)$. Then $H_{*}\left(\Omega S^{m-1} ; \mathbb{F}\right)$ is central in $H_{*}\left(\Omega \tau_{M, 0} ; \mathbb{F}\right)$ if and only if

(a) when $\mathbb{F}=\mathbb{F}_{2}, w_{m-1}\left(\tau_{M, 0}\right)=0$, or

(b) when $\frac{1}{2} \in \mathbb{F}, e\left(\tau_{M, 0}\right)=0$ where $e(\cdot)$ denotes the Euler class of $M$.

Proof. Consider the short exact sequence of algebras obtained from the collapsing Serre spectral sequence for $\Omega(p)$ :

$$
1 \rightarrow H_{*}\left(\Omega S^{m-1} ; \mathbb{F}\right) \rightarrow H_{*}\left(\Omega \tau_{M, 0} ; \mathbb{F}\right) \rightarrow H_{*}(\Omega M ; \mathbb{F}) \rightarrow 1 .
$$

Notice that $H_{*}\left(\Omega S^{m-1} ; \mathbb{F}\right)$ fails to be central if and only if there is an element $x$ which is a lift of an element $\bar{x}$ in $H_{*}(\Omega M ; \mathbb{F})$ and for which

$$
\left[\iota_{m-2}, x\right] \neq 0 \text {. }
$$

The coproduct of $\left[\iota_{m-2}, x\right]$ is given by

$$
\psi\left[\iota_{m-2}, x\right]=\Sigma( \pm 1)\left[\iota_{m-2}, x^{\prime}\right] \otimes x^{\prime \prime}+\Sigma( \pm 1) x^{\prime} \otimes\left[i_{m-2}, x^{\prime \prime}\right],
$$

where $\psi x=\Sigma x^{\prime} \otimes x^{\prime \prime}$. (This calculation is a straightforward exercise as in [4, p.216.) Thus if $x$ is an element of least degree such that $\left[\iota_{m-2}, x\right] \neq 0$, then $\left[\iota_{m-1}, x\right]$ is primitive; assume that $x$ has this property.

By the splitting $\Omega \tau_{m, 0} \rightarrow \Omega M \times \Omega S^{m-1}$, the module $\operatorname{Prim}_{*}\left(\Omega \tau_{M, 0} ; \mathbb{F}\right)$ of primitives for $H_{*}\left(\Omega \tau_{M, 0} ; \mathbb{F}\right)$, is given by

$$
\operatorname{Prim}_{*}(\Omega M ; \mathbb{F}) \oplus \operatorname{Prim}_{*}\left(\Omega S^{m-1} ; \mathbb{F}\right) .
$$

Notice that $\left[\iota_{m-2}, x\right]$ projects to zero in $H_{*}(\Omega M ; \mathbb{F})$, as $\Omega(p)_{*}\left(\iota_{m-2}\right)=0$. Thus $\left[\iota_{m-2}, x\right]$ must lie in $\operatorname{Prim}_{*}\left(\Omega S^{m-1} ; \mathbb{F}\right)$.

Hence $\left[\iota_{m-2}, x\right]$ is a scalar multiple of

1. $\left(\iota_{m-2}\right)^{p^{r}}$ for $m-1 \equiv 1(2)$ and $\mathbb{F}=\mathbb{F}_{p}$, 
2. 0 for $m-1 \equiv 1(2)$ with $\mathbb{F}=\mathbb{Q}$,

3. $\iota_{m-2}^{2^{r}}$ for $m-1 \equiv 0(2)$ and $\mathbb{F}=\mathbb{F}_{2}$,

4. $\left[\iota_{m-2}, \iota_{m-2}\right]^{p^{r}}$ for $m-1 \equiv 0(2)$ and $\mathbb{F}=\mathbb{F}_{p}$ for $p>2$, or

5. $\left[\iota_{m-2}, \iota_{m-2}\right]$ for $m-1 \equiv 0(2)$ and $\mathbb{F}=\mathbb{Q}$.

In any case, if $\left[\iota_{m-2}, x\right]$ is non-zero, then it follows that $\Omega(j)_{*}\left[i_{m-2}, x\right]$ has non-zero image in $H_{*}\left(\Omega E_{m} ; \mathbb{F}\right)$, as $\Omega E_{m}$ splits as $S O(m-1) \times \Omega S^{m-1}$, and the value of $\Omega(j)_{*}\left[i_{m-2}, x\right]$ projects to a non-zero element of $H_{*}\left(\Omega S^{m-1} ; \mathbb{F}\right)$. By these remarks, it suffices to calculate the value of $\Omega(j)_{*}(x)$ in the homology ring $H_{*}\left(\Omega E_{m} ; \mathbb{F}\right)$, and that will be addressed in several cases below.

By the formula

$$
[a b, z]=a[b, z] \pm[a, z] b
$$

(given in [4, p.216]), it may be assumed that $\Omega(j)_{*}(x)$ is indecomposable. Thus if $\left[\iota_{m-2}, x\right] \neq 0$ for $x$ of minimal degree, then $x$ has degree $m-2$ and $x$ projects to a non-zero algebra generator of $H_{*}(S O(m-1) ; \mathbb{F})$. Hence

1. $\Omega(j)_{*}(x)=x_{m-2}$, where $\left\langle\sigma^{*} w_{m-1}, x_{m-2}\right\rangle=1$ in $H^{*}\left(S O(m-1) ; \mathbb{F}_{2}\right)$, where $\sigma^{*}$ is the cohomology suspension, or

2. $\Omega\left(j_{*}\right)(x)=x_{m-2}$, where $\left\langle\sigma^{*} e_{m-1}, x_{m-2}\right\rangle \neq 0$, where $e_{m-1}$ is the Euler class in $H^{m-1}(B S O(m-1) ; \mathbb{F})$ with $\frac{1}{2} \in \mathbb{F}$.

Theorem 11.1 follows at once.

Theorem 11.1 will be used to identify the relation for $\left[B_{i, j}, m_{i}+m_{j}\right]$ in the loop space homology of $F(M, 2)$ under the restricted conditions that (1) the StiefelWhitney class and Euler class as described in the theorem vanish, and (2) the element $m$ is primitive. However, some additional topology is required, as follows.

It will be assumed throughout the rest of this section that $M=M^{\prime}-D$, where $D$ is a disk of positive radius in a manifold $M^{\prime}$ (or equivalently that $M$ is a $p$-manifold). Consider maps

$$
\gamma_{i, j}: \tau_{M, 0} \rightarrow F(M, k)
$$

defined by $\gamma_{i, j}(m, v)=\left(z_{1}, \ldots, z_{k}\right)$ with the points $z_{i}$ given as follows.

1. $m$ is an $M=M^{\prime}$-disk,

2. $v$ is a unit tangent vector at $m$,

3. $z_{i}=m$,

4. $z_{j}=\exp (v)$, where $\exp (\cdot)$ denotes the exponential map, and

5. $z_{t}=q_{t}$ for $t \notin\{i, j\}$, where $\left\{q_{1}, \ldots, q_{k}\right\}$ are $k$ distinct points in disk $-\{$ point $\}$.

Lemma 11.2. Assume that $M$ is a p-manifold.

1. The bundle projection $p: \tau_{M, 0} \rightarrow M$ has a cross-section $\sigma: M \rightarrow \tau_{M, 0}$. Thus if $M$ is simply-connected, the Serre spectral sequence with any field coefficients for the fibration $p: \tau_{M, 0} \rightarrow M$ collapses. 
2. There is a morphism of fibrations

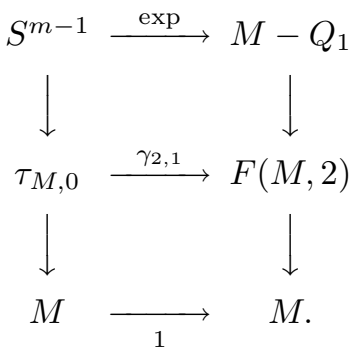

where the exponential map exp induces a split monomorphism in homology (as $M$ is a p-manifold).

3. The map $\Omega(\exp )$ sends the fundamental cycle $\iota$ in $H_{m-2}\left(\Omega S^{m-1} ; \mathbb{F}\right)$ to the element $B_{2,1}$ in $H_{m-2}(\Omega F(M, 2) ; \mathbb{F})$.

Proof. The existence of a section for the sphere bundle in the tangent bundle of $M$ follows at once since $M$ is a $p$-manifold. Since $M$ is simply-connected, the local coefficient system is trivial, and the Serre spectral sequence collapses from the existence of a section by inspection.

The morphism of fibrations in part (2) follows by definition of the map $\gamma_{2,1}$. The homological assertion in part (3) follows from the definitions.

Next, consider the composite

$$
M \stackrel{\sigma}{\longrightarrow} \tau_{M, 0} \stackrel{\gamma_{2,1}}{\longrightarrow} F(M, 2) \stackrel{\text { inclusion }}{\longrightarrow} M^{2} .
$$

denoted by $\Psi: M \rightarrow M^{2}$. Notice that this composite is homotopic to the diagonal map $M \rightarrow M^{2}$. This construction will be used to prove the next lemma.

Lemma 11.3. Let $M$ be a p-manifold $M$ where either

1. $\mathbb{F}=\mathbb{F}_{2}$ and $w_{m-1}\left(\tau_{M, 0}\right)=0$, or

2. $\frac{1}{2} \in \mathbb{F}$ and $e\left(\tau_{M, 0}\right)=0$,

If $m$ denotes a primitive homology class in $H_{*}(\Omega M ; \mathbb{F})$, then $\left[B_{2,1}, m_{2}+m_{1}\right]=0$ in $H_{*}(\Omega F(M, 2) ; \mathbb{F})$.

Proof. Consider the map $\gamma_{2,1}: \tau_{M, 0} \rightarrow F(M, 2)$. By Lemma 11.2(3),

$$
\Omega\left(\gamma_{2,1}\right)_{*}(\iota)=B_{2,1} \quad \text { in } H_{m-2}(\Omega F(M, 2) ; \mathbb{F}),
$$

where $\iota$ denotes the fundamental cycle in $H_{m-2}\left(\Omega S^{m-1} ; \mathbb{F}\right)$. By the hypotheses that either $w_{m-1}\left(\tau_{M, 0}\right)=0$ or $e\left(\tau_{M, 0}\right)=0$ together with Theorem 11.1, the element $\iota$ is central in $H_{*}\left(\Omega \tau_{M, 0} ; \mathbb{F}\right)$. Thus the element $B_{2,1}$ commutes with all elements in the image of the map

$$
H_{*}\left(\Omega \tau_{M, 0} ; \mathbb{F}\right) \stackrel{\gamma_{2,1_{*}}}{\longrightarrow} H_{*}(\Omega F(M, 2) ; \mathbb{F}) .
$$

Next, consider the cross-section $\sigma: M \rightarrow \tau_{M, 0}$ together with a primitive element $m$ in $H_{*}(\Omega M ; \mathbb{F})$. Since $m$ is primitive, and $\Omega(\Psi)_{*}(m)=m_{1} \otimes 1+1 \otimes m_{2}$ in $H_{*}\left(\Omega M^{2} ; \mathbb{F}\right)$, it follows that

$$
\Omega\left(\gamma_{2,1}\right)_{*}(m)=m_{1}+m_{2}+\text { "others" }
$$

where "others" is a primitive element in the ideal generated by $B_{2,1}$. Hence "others" is given by a scalar multiple of some power of the element $B_{2,1}$, say $\lambda B_{2,1}^{t}$. 
Since $B_{2,1}$ commutes with the image of $m$,

$$
\left[B_{2,1}, m_{1}+m_{2}+\lambda B_{2,1}^{t}\right]=0 .
$$

Thus

$$
\left[B_{2,1}, m_{1}+m_{2}\right]=0
$$

and the lemmas follows.

An immediate application of Lemma 11.3 is

Proposition 11.4. If $m$ denotes a primitive homology class in the loop space homology of a p-manifold $M$ where either

1. $\mathbb{F}=\mathbb{F}_{2}$ and $w_{m-1}\left(\tau_{M, 0}\right)=0$, or

2. $\frac{1}{2} \in \mathbb{F}$ and $e\left(\tau_{M, 0}\right)=0$,

then $\left[B_{i, j}, m_{i}+m_{j}\right]=0$ in the loop space homology of $F(M, k)$.

Proof. Notice that $\left[B_{i, j}, m_{i}+m_{j}\right]$ is obtained from the obvious permutation applied to the relation $\left[B_{2,1}, m_{2}+m_{1}\right]=0$. Thus Proposition 11.4 follows from 11.3.

Observe that Theorem 2.4.3(iii) and Theorem 2.10 follow from Proposition 11.4.

\section{On the Case of $M-$ P POINT $\}$ In $M$, Theorem 2.11}

A striking result of Félix and Thomas [16] is that the natural inclusion

$$
M-\{\text { point }\} \rightarrow M
$$

induces a surjection on loop space homology with rational coefficients when the following conditions are satisfied:

1. $M$ is 1-connected.

2. There exist at least 2 linearly independent algebra generators in $\bar{H}^{*}(M ; \mathbb{Q})$.

In this case, notice that $M-\{$ point $\}$ may be replaced by $M-\{$ disk $\}$ with $\left\{q_{1}, \ldots, q_{k}\right\}$ a set of $k$ distinct points in the disk with an interior point removed. Thus, define

$$
\phi: M-\{\text { disk }\} \rightarrow F(M, k)
$$

by the formula $\phi(m)=\left(m, q_{2}, q_{3}, \ldots, q_{k}\right)$. The inclusion $M-\{$ disk $\} \rightarrow M$ then factors as the composite given by the first coordinate projection map composed with $\phi, M-$ disk $\rightarrow F(M, k) \rightarrow M$.

Thus by [16] the composite

$$
\Omega(M-\text { disk }) \rightarrow \Omega F(M, k) \rightarrow \Omega M
$$

induces a surjection on rational homology whenever $M$ satisfies (i) and (ii). Furthermore, the Serre spectral sequence for $\Omega(p)$ collapses with any field coefficients if $\Omega(p)_{*}$ is surjective, as the fibration is multiplicative. The next lemma, as well as Theorem 2.11, follows.

Lemma 12.1. If $\Omega(M-$ disk $) \rightarrow \Omega(M)$ induces a surjection on $H_{*}(; \mathbb{F})$, then $H_{*}(\Omega F(M, k) ; \mathbb{F})$ is isomorphic to $\bigotimes_{i=0}^{k-1} H_{*}\left(\Omega\left(M-Q_{i}\right) ; \mathbb{F}\right)$ as a graded vector space. Furthermore, the natural inclusion $M-\{$ point $\} \rightarrow M$ induces a surjection map of Hopf algebras

$$
H_{*}\left(\Omega F\left(M-Q_{1}, k\right) ; \mathbb{F}\right) \rightarrow H_{*}(\Omega F(M, k) ; \mathbb{F})
$$


whenever $M$ satisfies the conditions of Félix and Thomas as listed above in (1) and (2).

\section{Proof of Theorem 2.12}

Notice that Theorem 2.12(1) is a restatement of a result of Félix and Thomas 17] described in the previous section.

To prove Theorem 2.12(2), observe that $M_{1} \vee M_{2}$ is a subspace of $M_{1} \times M_{2}$. Theorem 2.12(2) follows at once from the fact that $\Omega\left(M_{1} \vee M_{2}\right)$ is homotopy equivalent to a product where one factor is $\Omega\left(M_{1} \times M_{2}\right)$. A second proof is given by that of Theorem 2.1(4).

The proof of Theorem 2.12(3) is analogous to that of Theorem 2.12(1) and is omitted. Theorem 2.12(4) is the subject of sections 14 and 15. Finally, Theorem 2.12(5) follows because the induced maps of loop spaces fail to be surjective in homology by direct inspection. Specific computations are addressed in section 16 .

\section{ON $\Omega(G / H)$}

Consider

1. $G=S U(n)$ with subgroup $H=S U\left(n_{1}\right) \times \cdots S U\left(n_{k}\right)$ for $n_{1} \geq n_{j}$, or

2. $G=S p(n)$ with subgroup $H=S p\left(n_{1}\right) \times \cdots \times S p\left(n_{k}\right)$ for $n_{1} \geq n_{j}$.

Similar results will apply to $G=S O(n)$, but it is required that $n_{1}>n_{j}$ for $j \geq 2$, and these results will not be listed below. The calculations in this section will be used in section 15 .

Assume that $H \leq G$ with $H^{\prime}=S U\left(n_{1}\right)$ or $S p\left(n_{1}\right)$. Thus there is a commutative diagram

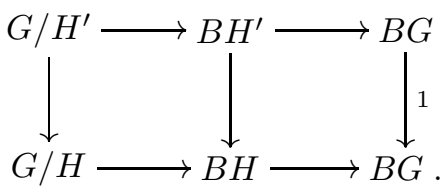

A computation of $H_{*}(\Omega(G / H) ; \mathbb{Z})$ follows at once from the next morphism of fibrations:

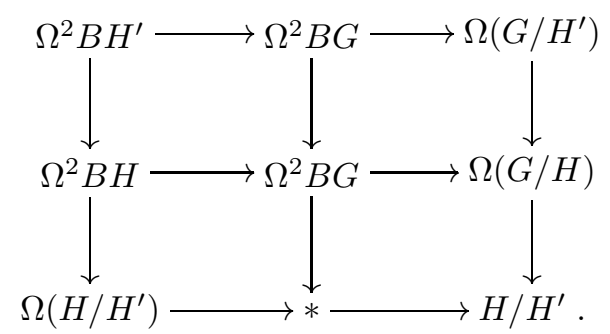

In particular, there is a multiplicative fibration

$$
\Omega\left(G / H^{\prime}\right) \rightarrow \Omega(G / H) \rightarrow H^{\prime \prime},
$$

where $H^{\prime \prime}$ denotes the topological group $H / H^{\prime}$. Notice that the homology of $H^{\prime \prime}$ as a ring is generated by classes of degree

1. at most $2 n_{1}-1$ if $G=S U(n)$, and

2. at most $4 n_{1}-1$ if $G=S p(n)$. 
The generators of $H_{*}\left(H^{\prime \prime} ; \mathbb{Z}\right)$ are infinite cycles in the integral homology Serre spectral sequence for

$$
\Omega\left(G / H^{\prime}\right) \rightarrow \Omega(G / H) \rightarrow H^{\prime \prime}
$$

by inspection. Since the spectral sequence is multiplicative, it collapses, and the next result follows.

Theorem 14.1. With the hypotheses above, there are isomorphisms

$$
H_{*}(\Omega(G / H) ; \mathbb{Z}) \cong H_{*}\left(H^{\prime \prime} ; \mathbb{Z}\right) \otimes H_{*}\left(\Omega\left(G / H^{\prime}\right) ; \mathbb{Z}\right)
$$

as $H_{*}\left(\Omega\left(G / H^{\prime}\right) ; \mathbb{Z}\right)$-modules. Furthermore, $H_{*}(\Omega(G / H) ; \mathbb{Z})$ is generated as an algebra by (1) the homology of its $(2 n-2)$-skeleton if $G=S U(n)$, or (2) the homology of its $(4 n-2)$-skeleton if $G=S p(n)$. Thus if $G$ is of rank at least 3, then $G / H$ is braidable.

Note: If $G / H$ is of rank 2 , then it is not braidable by Theorems 2.5 and 2.7. If $G / H$ is of rank 1 , it is evidently not braidable.

Proofs of the statements of Theorem 14.1 except for the statement concerning $G / H$ being braidable are sketched above. To prove the remaining statement, consider the case of $S U(n)$ first. There is a map $\Sigma \mathbb{C P}^{n-1} \rightarrow S U(n)$ which (1) is an embedding, and (2) the image in homology generates $H_{*}(S U(n) ; \mathbb{Z})$ as a ring.

Notice that $G / H^{\prime}$ is $2 n_{1}$-connected. Furthermore, the natural map $\Sigma \mathbb{C P}^{n_{j}-1} \rightarrow$ $S U\left(n_{j}\right)$ composed with the natural map

$$
S U\left(n_{j}\right) \rightarrow S U\left(n_{2}\right) \times \cdots \times S U\left(n_{k}\right) \rightarrow S U(n) / S U(n-1)
$$

is null and lifts to $\Omega(G / H)$.

Thus there are maps

$$
\mathbb{C P}^{n-1} / \mathbb{C P}^{n_{1}-1} \vee\left(\bigvee_{j=\ell}^{k} \Sigma \mathbb{C P}^{n_{j}-1}\right) \rightarrow \Omega(G / H),
$$

where the image in homology generates the integer homology ring of $\Omega(G / H)$. Passage to adjoints gives

$$
\Sigma\left(\mathbb{C P}^{n-1} / \mathbb{C P}^{n_{j}-1}\right) \vee\left(\bigvee_{j=2}^{k} \Sigma^{2} \mathbb{C P}^{n_{j}-1}\right) \rightarrow G / H
$$

Let $A$ denote the image of this last map. By cellular approximation, it can be assumed that $A$ is of dimension $2 n-1$. If $G / H$ is of dimension at least $4 n-1$, then the embedding of $A$ in $G / H$ is isotopic to an embedding with disjoint image in general position. Thus $G / H$ is braidable.

Similar arguments apply to the case of $G=S p(n)$, with $\mathbb{C} P^{n-1}$ being replaced by quasi-projective spaces.

Theorem 14.2. If $G=S U(n)$ with $H=S U\left(n_{1}\right) \times \cdots \times S U\left(n_{k}\right)$ for $n_{1} \geq n_{j}$ with dimension $(G / H) \geq 4 n-1$, then $G / H$ is braidable. Hence the commutativity relation $\left[m_{i}, m_{j}^{\prime}\right]=0$ holds in $H_{*} \Omega F(G / H, k)$. If $G=S p(n)$ with $H=S p\left(n_{1}\right) \times$ $\cdots \times S p\left(n_{k}\right)$ with dimension $(G / H) \geq 8 n-1$, then the analogous result holds. 
15. ON $\Omega F(M, k)$ FOR $M=S U(n), S p(n)$, OR $G / H$ PROOF OF THEOREM 2.13

The purpose of this section is to prove Theorem 2.13, which gives the structure for the loop space homology of $\Omega F(G, k)$ if $G$ is either $S U(n)$ or $S p(n)$, and coefficients are taken in the rational numbers. The main point here is that the structures for $G=S U(3)$ and $G=S p(2)$ are significantly different than those for $G=S U(n)$ or $G=S p(n-1)$ when $n \geq 4$. In addition, the results of section 14 will be applied in this section to the case of $\Omega F(M, k)$ for which $M$ is given by certain choices of homogeneous spaces.

Recall that by [14], any topological group with cardinality at least $k$ is a $\sigma_{k^{-}}$ manifold. Thus Theorem 2.1 applies to give a homotopy equivalence

$$
\Omega F(G, k) \longrightarrow \Omega G \times \Omega F\left(G-Q_{1}, k-1\right), \quad k \geq 2 .
$$

Restrict to coefficients in $\mathbb{Q}$ for the rest of this section, and assume that all spaces are localized at the rational numbers. Recall that the dimension of $G=S U(n)$ is $m=n^{2}-1$, while the dimension of $G=S p(n)$ is $m=2 n^{2}+n$.

In this case, the homotopy theoretic fibre (localized at the rational numbers) of the inclusion map $G-Q_{1}$ in $G$ is the half-smash product $S^{m-1} \triangleleft \Omega G$, where $m$ is the dimension $(G)$ [16]; the notation $X \triangleleft Y$ denotes the half-smash product $(X \times Y) /\{*\} \times Y$ for spaces $X$ and $Y$ with non-degenerate base-points $\{*\}$. Caution: This last equivalence for the homotopy theoretic fibre of the inclusion of $G-Q_{1}$ in $G$ is not an integral equivalence, nor even a $p$-local equivalence, in general.

Namely, the attaching map of the top cell in $G$ is in $\pi_{m-1}\left(G-Q_{1}\right)$, and the rational equivalence stated above follows at once from [16]. Notice that $G-Q_{i}$ is homotopy equivalent to $\left(G-Q_{1}\right) \vee \bigvee_{i-1} S^{m-1}$ for $i>1$. Thus the loop space homology of $G-Q_{i}$ is generated by the classes $B_{i, j}$ for $i>j \geq 1$ together with generators $x_{q}$ of degree $2 q$ for the loop space homology of $G$ as specified in section 14.

A more precise description is useful here. This description follows at one from [16]. The homotopy theoretic fibre, localized at the rational numbers, of

$$
\Omega\left(G-Q_{1}\right) \longrightarrow \Omega G
$$

is $\Omega\left(S^{q-1} \vee \Sigma^{q-1} \Omega G\right)$. A choice of map realizing this equivalence is multiplicative and is induced by the canonical multiplicative extension of (1) the adjoint of the attaching map of the top cell in $\mathrm{G}$, and (2) the Samelson product of this last map with a section for $\Omega\left(G-Q_{1}\right) \longrightarrow \Omega G$.

Theorem 2.4 applies to give the additive structure, and the multiplicative extensions are specified in the proof of Theorem 2.8.

Theorem 15.1. If all loop spaces are localized at the rational numbers, then there is a homotopy equivalence

$$
\Omega F(G, k) \longrightarrow(\Omega G)^{k} \times \Omega F\left(\mathbb{R}^{m}, k\right) \times \prod_{i=1}^{k-1} \Omega \Sigma\left[(\Omega G) \wedge\left(\Omega \bigvee_{i} S^{m-1}\right)\right]
$$

where $G$ is of dimension $n$.

The relations are specified in the proof of Theorems 2.4 and 2.8 as follows:

1. The infinitesimal braid relations are satisfied for all $B_{i, j}$ for $k \geq i>j \geq 1$.

2. $\left[B_{i, j}, m_{\ell}\right]=0$ for all $\ell \notin\{i, j\}$. 
3. If $G=S U(2)$ or $G=S p(1)$, then the homology ring of $\Omega F(G, k)$ is isomorphic to that of $\Omega(G) \times \Omega F\left(\mathbb{R}^{m}, k-1\right)$ as a Hopf algebra.

4. If $G=S U(3)$ or $G=S p(2)$, then $\left[x_{2}(i), x_{4}(j)\right]=B_{i, j}$ for $i>j$.

5. If $G=S U(n)$ or $G=S p(n-1)$ for $n \geq 4$, then $\left[x_{2 r}(i), x_{2 s}(j)\right]=0$ for all $i, j, r$, and $s$.

This last result together with Theorem 2.8 gives all of the relations in Theorem 2.13 , and thus all of the relations in the loop space homology with rational coefficients. Notice that the cases of rank one and rank two groups are different than those of rank at least three.

Similar results apply in case $G$ is replaced by the homogeneous spaces $M=G / H$ of section 14. If $G / H$ has rank at least two for its rational cohomology, then $G / H$ is an $e R$-manifold for $\mathrm{R}=\mathbb{Q}$ by Theorem 2.11. Thus the homology $\operatorname{ring}$ of $\Omega F(M, k)$ is isomorphic to

$$
H_{*}(\Omega(M) ; \mathbb{Q}) \otimes H_{*}\left(\Omega F\left(M-Q_{1}, k-1\right) ; \mathbb{Q}\right)
$$

as an $H_{*}\left(\Omega F\left(M-Q_{1}, k-1\right) ; \mathbb{Q}\right)$-module. The rest of the proof of the next theorem is that of the previous theorem together with the results of section 14 .

Theorem 15.2. If $G=S U(n)$ with $H=S U\left(n_{1}\right) \times \cdots \times S U\left(n_{k}\right)$ for $n_{1} \geq n_{j}$ with dimension $(G / H) \geq 4 n-1$, or if $G=S p(n)$ with $H=S p\left(n_{1}\right) \times \cdots \times S p\left(n_{k}\right)$ with dimension $(G / H) \geq 8 n-1$, then there is a rational homotopy equivalence

$$
\Omega F(G / H, k) \longrightarrow \Omega G / H \times \Omega F\left(G / H-Q_{1}, k-1\right), \quad k \geq 2 .
$$

The relations are specified in the proof of Theorems 2.4 and 2.8 as follows:

1. the infinitesimal braid relations are satisfied for all $B_{i, j}$ for $k \geq i>j \geq 1$,

2. $\left[B_{i, j}, m_{\ell}\right]=0$ for all $\ell \notin\{i, j\}$,

3. $\left[m_{i}, m_{j}^{\prime}\right]=0$ for $i>j$, and

4. $\left[B_{i, j}, m_{i}+m_{j}\right]=0$ for $i>j$.

\section{Special Cases; Theorem 2.14}

The purpose of this section is to provide information concerning $\Omega F(M, k)$ for the special cases $M=S^{n}, \mathbb{R P}^{n}, \mathbb{C P}^{n}$, or $\mathbb{H P}^{n}$. Recall that there are classical principal $G$-bundles

$$
\begin{aligned}
S^{n} \rightarrow \mathbb{R P}^{n} & \text { with } G=\mathbb{Z} / 2 \mathbb{Z}, \\
S^{2 n+1} \rightarrow \mathbb{C P}^{n} & \text { with } G=S^{1}, \\
S^{4 n+3} & \rightarrow \mathbb{H P}^{n} \quad \text { with } G=S^{3} .
\end{aligned}
$$

One of Xicoténcatl's [31 results is stated next: Let $G$ act on a space $Y$ with $Y \rightarrow Y / G$ a principal $G$-bundle. Then there is a principal $G^{k}$-bundle

$$
F_{G}(Y, k) \rightarrow F(Y / G, k)
$$

where $F_{G}(Y, k)=\left\{\left(y_{1}, \ldots, y_{k}\right) \in Y^{k} \mid y_{i} G \cap y_{j} G=\varnothing\right.$ if $\left.i \neq j\right\}$. Further, in the case of the above fibrations, he obtains the following splitting result in [31]: 
Theorem 16.1. There are homotopy equivalences

$$
\begin{aligned}
& \Omega F\left(\mathbb{R P}^{n}, k\right) \rightarrow(\mathbb{Z} / 2 \mathbb{Z})^{k} \times \Omega F_{\mathbb{Z} / 2 \mathbb{Z}}\left(S^{n}, k\right), \\
& \Omega F\left(\mathbb{C P}^{n}, k\right) \rightarrow\left(S^{1}\right)^{k} \times \Omega F_{S^{1}}\left(S^{2 n+1}, k\right), \\
& \Omega F\left(\mathbb{H} \mathbb{P}^{n}, k\right) \rightarrow\left(S^{3}\right)^{k} \times \Omega F_{S^{3}}\left(S^{4 n+1}, k\right) .
\end{aligned}
$$

In the case of $S^{n}$, one has

Theorem 16.2. $\quad$ 1. If $n \geq 1$, then $\Omega F\left(S^{2 n+1}, k\right)$ is homotopy equivalent to

$$
\Omega S^{2 n+1} \times \Omega F\left(\mathbb{R}^{2 n+1}, k-1\right) .
$$

2. The space $F\left(S^{n}, 3\right)$ is homotopy equivalent to $S O(n+1) / S O(n-1)$.

3. The natural composite $S O(n+1) \rightarrow F\left(S^{n}, k\right) \rightarrow F\left(S^{n}, 3\right)$ induces a surjection on the level of mod-2 loop space homology for $n \equiv 0(2)$ and $n>2$.

4. There is a homotopy equivalence

$$
F\left(S^{2}, k\right) \rightarrow S O(3) \times F\left(S^{2}-Q_{3}, k-3\right) .
$$

Remark 16.3. Theorem 16.2(4) is classical and has appeared in several contexts, one of which is [14].

Proof. Let $\mathbb{Z} / k \mathbb{Z}$ act freely on $S^{2 n+1}$, where $\tau$ is a generator for $\mathbb{Z} / k \mathbb{Z}$. A section for the first coordinate projection map $\pi: F\left(S^{2 n+1}, k\right) \rightarrow S^{2 n+1}$ is given by $\sigma(z)=\left(z, \tau z, \tau^{2} z, \ldots, \tau^{k-1} z\right)$. Thus part (1) follows. Parts 2 and 4 are standard.

A map $\gamma: S O(n+1) \rightarrow F\left(S^{n}, k\right)$ is gotten by sending $\lambda$ to $\left(\lambda\left(q_{1}\right), \cdots, \lambda\left(q_{k}\right)\right)$, where $\left\{q_{1}, \ldots, q_{k}\right\}$ is a set of $k$ distinct points in $S^{n}$. The composite is induced by

$$
S O(n+1) \rightarrow S O(n+1) / S O(n-1) \rightarrow F\left(S^{n}, 3\right),
$$

as $\left(q_{1}, q_{2}, q_{3}\right)$ may be chosen to be an orthonormal 3-frame. The map $S O(n+1) \rightarrow$ $S O(n+1) / S O(n-1)$ surjects on the level of mod-2 loop space homology, and the result follows.

\section{REFERENCES}

[1] M. Bendersky, and S. Gitler, The Cohomology of Certain Function Spaces, Trans. A.M.S., 326, No. 1, (1991), 423-440. MR 93d:55005

[2] V. Chari, and A. Pressley, A Guide to Quantum Groups, Cambridge University Press, 1998, Cambridge, England. MR 95j:17010 (1st ed.)

[3] D. Cohen, F. R. Cohen, and M. Xicoténcatl, Lie Algebras Associated to Fiber-Type Arrangements, submitted.

[4] F. R. Cohen, The homology of iterated loop spaces, Springer-Verlag Lecture Notes in Mathematics, 533(1976), 207-351. MR 55i:9096

[5] F. R. Cohen, On genus one mapping class groups, function spaces, and modular forms, to appear in Cont. Math..

[6] F. R. Cohen, S. Gitler, Loop spaces of configuration spaces, braid-like groups, and knots, in Cohomological Methods in Homotopy Theory, Birkhäuser Verlag Progress in Mathematics vol. 196(2001), Basel, Berlin, Boston, 59-78.

[7] F. R. Cohen, J. C. Moore, and J. A. Neisendorfer, Torsion in homotopy groups, Ann. of Math. 109(1979), 121-168. MR 80e:55024

[8] F. R. Cohen and T. Sato, On groups of homotopy groups, loop spaces, and braid-like groups, preprint.

[9] F. R. Cohen and L. R. Taylor, (a) On the representation theory associated to the cohomology of configuration spaces, Cont. Math. 146(1993), 91-109. MR 94i:57057

(b) Gelfand-Fuks cohomology, the cohomology of function spaces and the cohomology of configuration spaces, Springer-Verlag Lecture Notes in Mathematics, 657 (1979), 106-143. MR 80f:58050 
[10] F. R. Cohen, and M. Xicoténcatl, On orbit configuration spaces associated to the Gaussian integers: homotopy groups, and homology groups, to appear in a Special Issue of Topology and its Applications devoted to the meeting "Arrangements in Boston", to appear.

[11] V. G Drinfel'd, On quasi-triangular quasi-Hopf algebras, and a certain group closely connected with Gal( $(\overline{\mathbb{Q}} / Q)$, Leningrad Math. J., 2(1991), 829-60. MR 92f:16047

[12] V. G. Drinfel'd, On the structure of quasi-triangular quasi-Hopf algebras, Funct. Anal. Appl. 26(1992), 63-5. MR 93i:16052

[13] E. Fadell and S. Husseini, (a) The space of loops on configuration spaces and the MajerTerracini index, Topological Methods in Nonlinear Analysis, Journal of the Julius Schauder Center 11 (1998), 249-271. MR 2000a:55020 (b) Geometry and Topology of Configuration Spaces, Springer-Verlag, Springer Monographs in Mathematics, (2001). CMP 2001:06

[14] E. Fadell and L. Neuwirth, Configuration spaces, Math. Scand. 10 (1962), 119-126. MR 25:4537

[15] M. Falk, and R. Randell, The lower central series of a generalized pure braid arrangement, Invent. Math. 82 (1985),77-88. MR 88c:20048

[16] Y. Félix and J.-C. Thomas, Effet d'un attachement cellulaire dans l'homologie de l'espace des lacets, Ann. Inst. Fourier, Grenoble 39, 1(1989), 207-224. MR 99j:55012

[17] Y. Félix, and J.C. Thomas, Homologie des espaces de lacets des espaces de configuration, Ann. Inst. Fourier (Grenoble) 44 (1994), no. 2, 559-568. MR 95i:55007

[18] T. Ganea, A generalization of the homology and homotopy suspension, Comment. Math. Helv. 39 (1965) 295-322. MR 31:4033

[19] P. Hilton, On the homotopy groups of a union of spheres, Comment. Math. Helv. 29 (1955), 59-92. MR 16:1043d

[20] N. Jacobson, Lie algebras, Interscience Press, 1962. MR 26:1345

[21] T. Kohno, Linear representations of braid groups and classical Yang-Baxter equations, Cont. Math. 78 (1988), 339-363. MR 90h:20056

[22] T. Kohno, Vassiliev invariants, and the de Rham complex on the space of knots, Cont. Math. 179(1994),123-138. MR 96g:57010

[23] I. Kriz, On the rational homotopy type of configuration spaces, Ann. of Math. 139(1994), 227-237. MR 95c:55012.

[24] J. Milnor, On the construction $F[K]$, London Mathematical Society Lecture Notes, 4(1972),119-136.

[25] J. Milnor, and J. Moore, On the structure of Hopf algebras, Ann. of Math., 81 (1965), 211-264. MR 30:4259

[26] J. Milnor and J. Stasheff, Characteristic classes, Ann. of Math. Studies 76, Princeton University Press, Princeton, 1974. MR 55:13428

[27] N. Steenrod, The Topology of Fibre Bundles, Princeton University Press, Princeton, 1951. MR 12:522b

[28] R. Thom, Quelques propriétés globales des variétés différentiables, Comment. Math. Helv. 28 (1954), 17-86. MR 15:890b

[29] B. Totaro, Configuration spaces of algebraic varieties, Topology 35 (1996), 1057-1067. MR 97g:57033

[30] G. W. Whitehead, Elements of Homotopy Theory, Springer-Verlag Graduate Texts in Mathematics, 61(1978). MR 80b:55001

[31] M. Xicoténcatl, Orbit configuration spaces, infinitesimal braid relations, and equivariant function spaces, Trans. A.M.S., to appear.

Department of Mathematics, University of Rochester, Rochester, New York 14627

E-mail address: cohf@math.rochester.edu

Department of Mathematics, University of Rochester, Rochester, New York 14627, and Departamento de Matemáticas, Cinvestav, Apdo. Postal 14-740, México, D.F. 07300

E-mail address: sgitler@math.cinvestav.mx 